\title{
Effect of Urea on Solvation Dynamics and Rotational Relaxation of Coumarin 480 in Aqueous Micelles of Cationic Gemini Surfactants with Different Spacer Groups
}

\author{
Sunita Kumari, ${ }^{\dagger}$ Sonu, ${ }^{\dagger}, \S$ Sayantan Halder, ${ }^{\dagger}$ Rishika Aggrawal, ${ }^{\dagger}$ Ganapathisubramanian Sundar, ${ }^{*,+}$ \\ and Subit K. Saha* $* \dagger$
}

${ }^{\dagger}$ Department of Chemistry, Birla Institute of Technology \& Science (BITS), Pilani, Pilani Campus, Pilani, Rajasthan 333031, India

${ }^{\ddagger}$ Department of Chemistry, Birla Institute of Technology \& Science (BITS), Pilani, Hyderabad Campus, Hyderabad, Telangana 500078, India

\section{Supporting Information}

ABSTRACT: The present work highlights the effect of urea on solvation dynamics and the rotational relaxation of Coumarin 480 (C-480) in the Stern layer of aqueous micelles of cationic gemini surfactants, $12-4(\mathrm{OH})_{n}-12(n=0,1,2)$. UV-visible absorption, steady-state fluorescence and fluorescence anisotropy, time-resolved fluorescence and fluorescence anisotropy, and dynamic light scattering measurements have been carried out for this study. The formation of micelles becomes disfavored in the presence of urea at high concentration. Solvation dynamics is bimodal in nature with fast solvation as a major component. The average solvation time increases, reaches a maximum, and then decreases with increasing concentration of urea because the degree of counterion dissociation also follows the same order with the addition of urea in the micellar

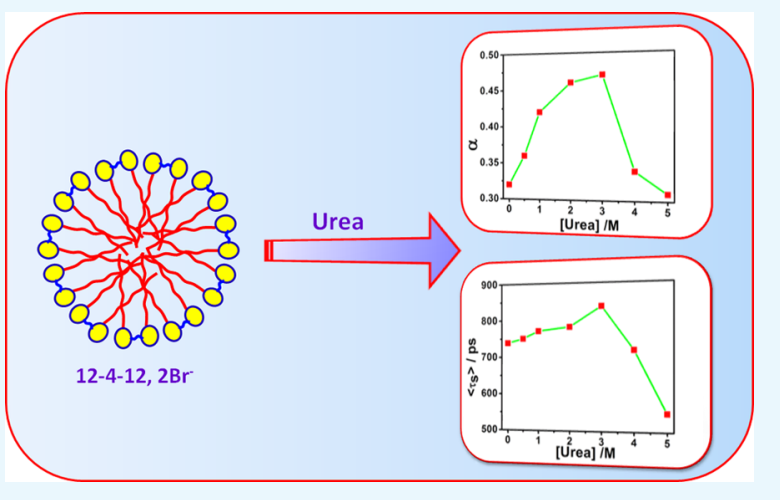
solution. With increased degree of counterion dissociation, the extent of clustering of water molecules is increased, resulting in slower solvation process. The $-\mathrm{OH}$ group present in the spacer group of gemini surfactant controls the rate of solvation by shielding the water molecules from the probe molecules forming hydrogen bond. The microviscosity of micelles is decreased with increasing concentration of urea, as a result of which the rotational relaxation process becomes faster. In the presence of the $-\mathrm{OH}$ group in the spacer group, the microviscosity of micelles is enhanced, resulting in longer rotational relaxation time. Rotational relaxation process is bimodal in nature with the major contribution from the fast component to the fluorescence depolarization. Slow rotational relaxation is mainly due to the lateral diffusion of C-480 molecules along the surface of the micelle. The tumbling motion of the micelle as a whole is much slower than the lateral diffusion of C-480. Wobbling motion of $\mathrm{C}-480$ becomes faster with increasing concentration of urea as a result of decreased microviscosity of micelles. The alignment of C-480 molecules in micelles might change with changing microviscosity.

\section{INTRODUCTION}

Micelles, the assemblies of surfactant molecules, up to certain extent mimic the environment of lipid bilayers. The studies on micellar organization and dynamics are paid special attention because the general principle for the micelles formation is related to other molecular assemblies, such as bilayers, reverse micelles, liposomes, and biological membrane. ${ }^{1-4}$ For biophysical or structure biological studies, surfactants are used to maintain the purified protein in its native and functional states. ${ }^{5,6}$ The presence of an additive modifies various aggregating properties of the micellar solution of surfactant. ${ }^{7-11}$ Urea, a well-known protein denaturant, with ionic and nonionic surfactants increases the critical micellar concentration $(\mathrm{cmc})$ values, ${ }^{12-16}$ reduces the aggregation number of micelles, ${ }^{17,18}$ etc. The solubility of hydrocarbons in aqueous medium is enhanced in the presence of urea. Mixtures of surfactant and urea are used for various applications in membrane-protein research. ${ }^{19-23}$ Urea facilitates the transfer of purified membrane protein from detergent micelles to lipid bilayers. ${ }^{23}$ Two different mechanisms are proposed regarding urea-induced protein denaturation. ${ }^{24-27}$ In one case, urea acts as a water structure breaker or "chaotrope" by facilitating the solvation of nonpolar solute. In another case, urea weakens the intramolecular proteinous bond on directly binding to proteins. $^{24-27}$ Various studies have been carried out to understand the properties of urea-water system. Few studies reported that urea acts as a water "structure breaker", 28,29 whereas other studies showed that urea acts as a water "structure maker". ${ }^{30,31}$ Some other studies showed the combination of these two effects in urea. ${ }^{32,33}$ Hayashi et al. ${ }^{34}$ showed that urea retains the weakly associated water in the tetrahedral structure

Received: November 8, 2017

Accepted: February 27, 2018

Published: March 14, 2018 
and thus it is not a strong structure breaker of water. Recently, Choudhury et al. have reported that urea can substitute water in the hydrogen-bonded network without breaking its tetrahedral, hydrogen-bonded structure. ${ }^{35}$

Water is a very important part of all biological systems. In these systems, water may exist in free and bound states. ${ }^{36}$ The study of water dynamics in organized assemblies possesses immense significance. It is known that the properties of solvent surrounding the reactants present in different biological systems and biomimicking organized assemblies significantly control various chemical reactions, such as charge transfer, electron transfer, etc. ${ }^{7,8,37}$ Thus, the study of the physicochemical properties of a confined medium surrounding probe molecules needs to be carried out to understand and control various processes. The study of solvation dynamics provides us with the information regarding the behavior of water molecules surrounding probe molecules, giving in-depth knowledge about the microenvironment. Pure water exhibits very fast solvation dynamics. In organized assemblies, solvation dynamics retarded many folds compared to that in the bulk. ${ }^{36}$ Many techniques have been used to study the dynamics of water in water-urea mixture. Infrared pump-probe spectroscopy has been used to study the effect of urea on the structure and dynamics of water, and it was found that even at high concentration of urea, dynamics of water has not been altered. ${ }^{38}$ Only a small fraction of water molecules displays slower dynamics than bulk water molecules by forming specific water-urea complex. Idrissi et al. ${ }^{39}$ studied the shorttime dynamics of water-urea mixture and the results showed that addition of urea leads to an overall isotropy and stiffening of the short-time dynamics of both the species. Many of these studies concentrated on the study of water dynamics in waterurea mixture. ${ }^{38-41}$ Few reports are available on the study of urea dynamics as a function of urea concentration in water-urea mixture. ${ }^{42,43}$ The effect of urea concentration on the dynamics of water in organized assemblies of surfactant molecules is scarcely studied. Hazra et al. studied the dynamics of water, urea, and water-urea mixture inside the reverse micelles of anionic surfactant. ${ }^{44}$ Chattopadhyay et al. studied the effect of urea on the organization and dynamics of Triton X-100 micelles. ${ }^{16}$ Ruiz et al. studied the microenvironmental properties of sodium dodecyl sulfate in aqueous urea solution. ${ }^{4}$

Nowadays, a class of surfactants called as "gemini surfactant" is attracting much interest in materials science, biological sciences, nanotechnology, supramolecular chemistry, etc. Gemini surfactants are a special class of surfactants comprising two hydrophobic tails and hydrophilic headgroups covalently connected by a spacer group at their headgroups. ${ }^{46}$ Some of the physical properties of gemini surfactants are far more superior to those of their conventional counterparts. ${ }^{46}$ Different groups have studied the effect of urea on various physical properties of conventional surfactants. Dey et al. studied the effects of urea on the aggregation properties of chiral surfactants. ${ }^{47}$ Keller et al. ${ }^{48}$ reported the impact of urea on nonionic sugar-based surfactants. The effect of urea on the aggregation behavior of gemini surfactants and their mixed micelles with pluronic surfactant was reported by Mahajan et al. ${ }^{49}$ Studies on the effect of urea concentration on the solvation dynamics in the micellar environment are scarce. Considering the importance of gemini surfactants over conventional surfactants and urea in the protein denaturation process, it would be valuable to study the effect of urea on the solvation and rotational relaxation dynamics in aqueous micelles of gemini surfactant. If there is any effect of urea on the micellization behavior of gemini surfactants with different spacer groups, then how does it affect the solvation dynamics and rotational relaxation processes of the probe molecule? Whether the water structure breaker or maker properties of urea or some other factor(s) guide(s) the rate of solvation process? Is the effect of urea on the solvation dynamics and rotational relaxation and micellization behavior of gemini surfactant with different spacer groups same or different? Answers to these questions are important as surfactant-urea mixtures are used in various membrane-protein research works. ${ }^{19-23}$

Recently, we have studied the solvation dynamics in the presence of pure gemini surfactants and mixed micelles of gemini surfactant and conventional surfactants. ${ }^{50-53}$ In the present study, we have investigated the effect of urea concentration on the aggregation properties of gemini surfactants with different spacer groups (with and without $-\mathrm{OH}$ group $(\mathrm{s})$ ) and also its effect on the solvation dynamics and rotational relaxation of Coumarin 480 (C-480) in aqueous micelles of those surfactants (Scheme 1). To probe the solvation dynamics, the solute chosen

Scheme 1. Molecular Structures of Gemini Surfactants and Coumarin 480

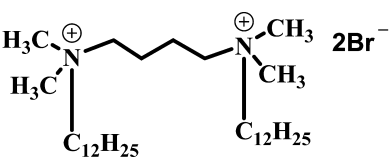

12-4-12 (Gemini-A)<smiles></smiles>

$12-4(\mathrm{OH})_{2}-12($ Gemini-C)<smiles>CCCCCCC(O)CC(C)(C)N(C)C</smiles>

12-4(OH)-12 (Gemini-B)

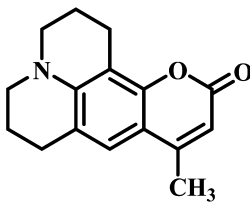

Coumarin 480 (C-480) should have zero or low dipole moment in the ground state and very high dipole moment in the excited state, ${ }^{36}$ as C-480 is such kind of molecule. Moreover, because of the presence of several basic centers, it can form hydrogen bonds. ${ }^{54-59}$ There are arguments on the cleavage of hydrogen bond upon excitation. ${ }^{56,58}$ However, a more convincing report by Zhao et al. ${ }^{59}$ shows that the early time of photoexcitation of C-480 to the excited state is strengthened due to the intermolecular hydrogen bonding between C-480 and hydrogen-bond-donating solvents/ groups. The surfactants used in the present study have been explored before to demonstrate the effect of $-\mathrm{OH}$ group $(\mathrm{s})$ on solvation dynamics. ${ }^{50}$ To the best of our knowledge, the solvation dynamics and rotational relaxation in the mixed systems of urea and gemini surfactants with different spacer groups have not been reported to date. The microenvironmental properties of C-480 within the micelles of gemini surfactants with varying urea concentration have been studied. Two-step and wobbling-in-a-cone models have been explored to show the bimodal behavior of rotational relaxation processes and to demonstrate the wobbling motions in the micelles. Results obtained from this study could be useful to understand the water dynamics in biological systems in the presence of urea. 


\section{RESULTS AND DISCUSSION}

2.1. Effect of Urea on cmc. Cmc and degree of counterion dissociation $(\alpha)$ values of all studied gemini surfactants in the absence and presence of various concentrations of urea have been determined by conductivity measurements performed at 298.15 K following Williams's method. ${ }^{60}$ A plot of specific conductivity, $\kappa$, versus concentration of surfactant with varying concentrations of urea for 12-4-12 is given in Figure 1 as a

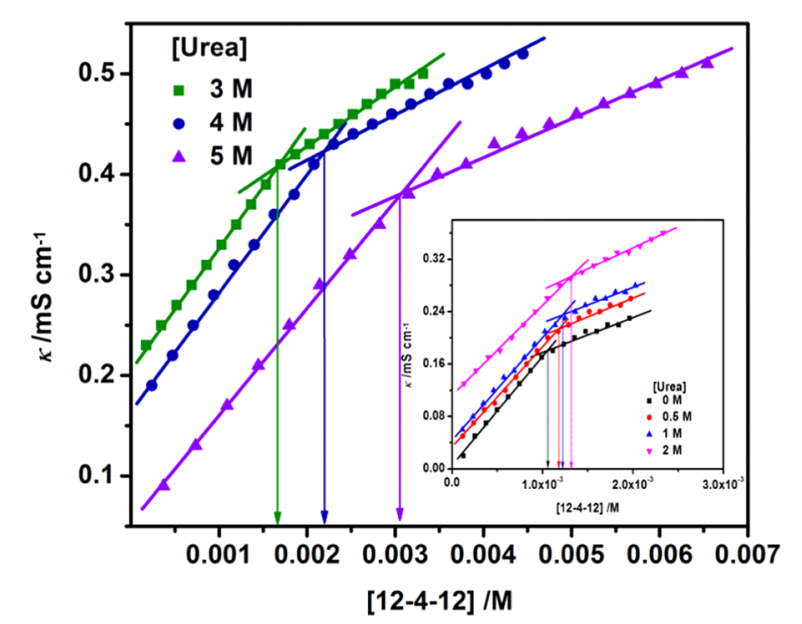

Figure 1. Plot of $\kappa$ vs concentration of 12-4-12 with varying concentrations of urea at $298.15 \mathrm{~K}$.

representative one. Similar changes in $\kappa$ with change in concentration of surfactant at varying concentrations of urea have been noted in cases of other two surfactants as well. Although a cmc value has been calculated from the break point of two intersecting lines, a value of $\alpha$ has been calculated by calculating the ratio of the slopes of the straight lines in the postmicellar region to the premicellar region. ${ }^{61}$ The values of cmc obtained are given in Table 1, and the values of $\alpha$ are given later (Table 4), where relevant discussion has been made. Cmc values of all of the pure gemini surfactants are in good agreement with the reported values, ${ }^{50,62}$ indicating the goodness of $\mathrm{cmc}$ values in the presence of urea as well. It has been noted that the $\mathrm{cmc}$ value of gemini surfactants enhances with increasing concentration of urea. It is reported in the literature that the solubility of a surfactant increases many folds in the presence of urea. ${ }^{63}$ Increased solubility of surfactant ions in the solution, i.e., their reduced solvophobicity, delays the aggregation process of surfactant molecules. In other words, the hydrophobic interactions between surfactant molecules are decreased in the presence of urea. ${ }^{39}$ Consequently, the concentration at which surfactant molecules start to form aggregates is increased.

To support the increment in $\mathrm{cmc}$ value with increasing concentration of urea in aqueous phase, the mole fraction partition coefficient of gemini surfactants from aqueous phase to micellar phase $\left(K^{\mathrm{mic}}\right)$ has been determined using eq 1 for all micellar systems ${ }^{48}$

$$
K^{\mathrm{mic}}=\frac{55.5 \mathrm{M}}{\mathrm{cmc}}
$$

where $55.5 \mathrm{M}$ is the molar concentration of water. The $K^{\text {mic }}$ values calculated are given in Table 1 , which are found to be decreased with increasing concentration of urea. In the presence of urea, because of decrease in the water-to-micelles partition coefficient, the cmc value of a gemini surfactant is increased. The values of standard molar Gibbs free energy of micellization have been calculated using eq $2^{48}$

$$
\Delta G^{\mathrm{o} \text {,mic }}=-R T \ln K^{\mathrm{mic}}
$$

$\Delta G^{\circ \text {,mic }}$ values at $298.15 \mathrm{~K}$ and various urea concentrations are given in Table 1, which are found to be increased with increasing concentration of urea. This also indicates that the micellization process becomes less favorable with increasing urea concentration.

2.2. UV-Visible Absorption and Steady-State Fluorescence Study. UV-visible absorption and steady-state fluorescence spectra of C-480 have been obtained in the presence of pure gemini surfactants and also in the presence of gemini surfactant at various concentrations of urea. Figure 2

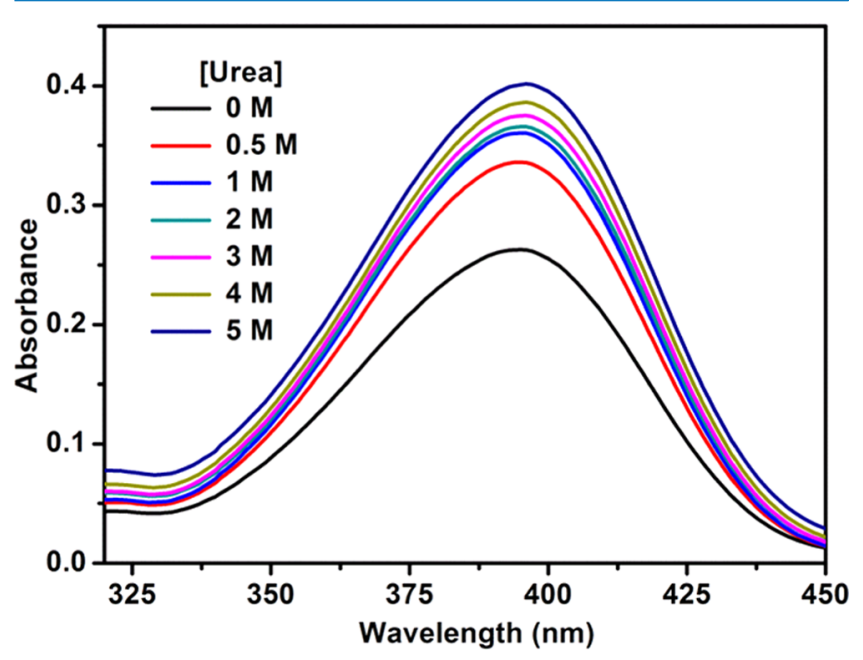

Figure 2. Absorption spectra of C-480 in the presence of pure 12-4-12 and 12-4-12 at varying concentrations of urea. [C-480] $=5 \mu \mathrm{M}$.

Table 1. Critical Micelle Concentration $(\mathrm{cmc})$, Mole Fraction Partition Coefficient $\left(K^{\mathrm{mic}}\right)$, and Standard Molar Gibbs Free Energy

\begin{tabular}{|c|c|c|c|c|c|c|c|c|c|}
\hline $\begin{array}{l}\text { urea } \\
(\mathrm{M})\end{array}$ & $\begin{array}{l}\text { Gemini-A } \\
\mathrm{cmc}(\mathrm{mM})\end{array}$ & $\begin{array}{l}\text { Gemini-B } \\
\mathrm{cmc}(\mathrm{mM})\end{array}$ & $\begin{array}{l}\text { Gemini-C } \\
\mathrm{cmc}(\mathrm{mM})\end{array}$ & $\begin{array}{l}\text { Gemini-A } \\
K^{\text {mic }}\left(10^{4}\right)\end{array}$ & $\begin{array}{l}\text { Gemini-B } \\
K^{\text {mic }}\left(10^{4}\right)\end{array}$ & $\begin{array}{l}\text { Gemini-C } \\
K^{\text {mic }}\left(10^{4}\right)\end{array}$ & $\begin{array}{l}\text { Gemini-A } \Delta G^{\circ, \text { mic }} \\
\quad\left(\mathrm{kJ} \mathrm{mol}{ }^{-1}\right)\end{array}$ & $\begin{array}{l}\text { Gemini-B } \Delta G^{\circ, \text { mic }} \\
\quad\left(\mathrm{kJ} \mathrm{mol}{ }^{-1}\right)\end{array}$ & $\begin{array}{c}\text { Gemini-C } \\
\Delta G^{\circ, \text { mic }} \\
\left(\mathrm{kJ} \mathrm{mol}^{-1}\right)\end{array}$ \\
\hline 0.0 & $1.06 \pm 0.03$ & $0.96 \pm 0.03$ & $0.88 \pm 0.02$ & $5.24 \pm 0.15$ & $5.78 \pm 0.17$ & $6.31 \pm 0.14$ & $-26.93 \pm 0.07$ & $-27.18 \pm 0.07$ & $-27.40 \pm 0.05$ \\
\hline 0.5 & $1.18 \pm 0.04$ & $1.07 \pm 0.03$ & $0.96 \pm 0.03$ & $4.70 \pm 0.15$ & $5.19 \pm 0.14$ & $5.78 \pm 0.17$ & $-26.67 \pm 0.07$ & $-26.91 \pm 0.07$ & $-27.18 \pm 0.07$ \\
\hline 1.0 & $1.23 \pm 0.03$ & $1.21 \pm 0.03$ & $1.17 \pm 0.03$ & $4.51 \pm 0.11$ & $4.59 \pm 0.11$ & $4.74 \pm 0.11$ & $-26.57 \pm 0.06$ & $-26.61 \pm 0.06$ & $-26.69 \pm 0.05$ \\
\hline 2.0 & $1.31 \pm 0.05$ & $1.47 \pm 0.07$ & $1.67 \pm 0.09$ & $4.24 \pm 0.16$ & $3.78 \pm 0.18$ & $3.32 \pm 0.17$ & $-26.41 \pm 0.09$ & $-26.12 \pm 0.12$ & $-25.81 \pm 0.13$ \\
\hline 3.0 & $1.66 \pm 0.08$ & $1.87 \pm 0.08$ & $1.78 \pm 0.11$ & $3.34 \pm 0.15$ & $2.97 \pm 0.12$ & $3.12 \pm 0.18$ & $-25.82 \pm 0.11$ & $-25.53 \pm 0.11$ & $-25.65 \pm 0.15$ \\
\hline 4.0 & $2.19 \pm 0.10$ & $2.12 \pm 0.11$ & $2.02 \pm 0.12$ & $2.53 \pm 0.11$ & $2.62 \pm 0.13$ & $2.75 \pm 0.16$ & $-25.14 \pm 0.11$ & $-25.22 \pm 0.13$ & $-25.34 \pm 0.15$ \\
\hline 5.0 & $3.05 \pm 0.12$ & $2.63 \pm 0.12$ & $2.46 \pm 0.10$ & $1.82 \pm 0.07$ & $2.11 \pm 0.09$ & $2.26 \pm 0.09$ & $-24.31 \pm 0.09$ & $-24.68 \pm 0.11$ & $-24.85 \pm 0.11$ \\
\hline
\end{tabular}
of Micellization $\left(\Delta G^{\circ, \text { mic }}\right)$ of Gemini Surfactants in the Presence of Urea 
depicts the absorption spectra of C-480 in the presence of $10 \mathrm{mM}$ 12-4-12 and 12-4-12 (10 mM) at various concentrations of urea as a representative. Figure 3 demonstrates the fluorescence

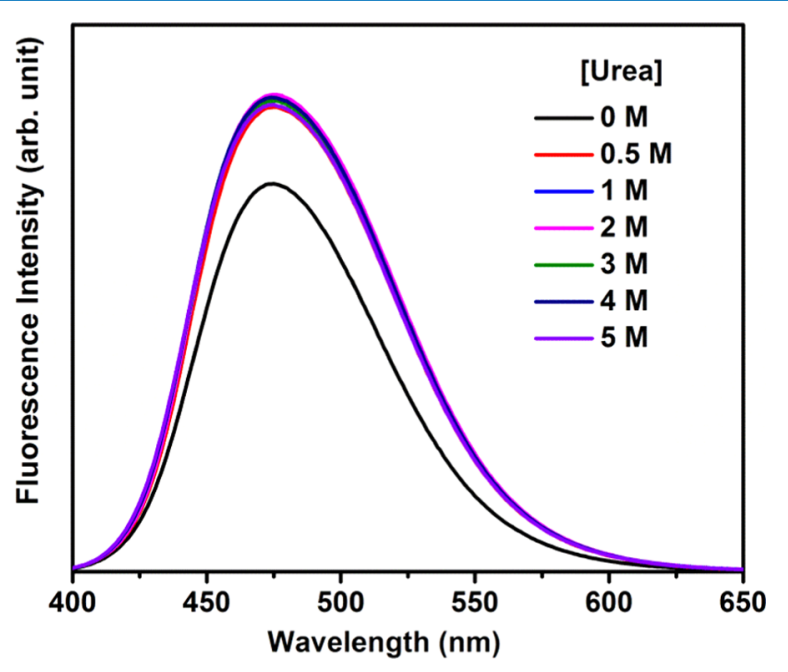

Figure 3. Fluorescence spectra of C-480 in the presence of pure 12-4-12 and 12-4-12 at varying concentrations of urea. [C-480] $=5 \mu \mathrm{M}$.

spectra of C-480 in the presence of pure $10 \mathrm{mM} \mathrm{12-4-12}$ and 124-12 (10 mM) at various concentrations of urea. Similar absorption and fluorescence spectra are observed in the presence of each of the other two gemini surfactants and urea systems as well. In both $10 \mathrm{mM}$ Gemini-A and Gemini-B, the absorption and fluorescence peak maxima of C-480 are 395 and $475 \mathrm{~nm}$, respectively. In $10 \mathrm{mM}$ Gemini-C, these values are 393 and 474 $\mathrm{nm}$, respectively. There is no significant change in peak maxima values upon addition of urea. Absorption and fluorescence peak maxima values of C-480 in all of the studied systems are given in Table S1. It is noteworthy that an increase in the absorbance and fluorescence intensity with increasing concentration of urea has been observed in pure water as well (spectra not shown). It could be because of either the effect of urea on hydrogen bonding between C-480 and water and/or the salting-in effect of urea (a thorough study will be done later). However, this effect can be ruled out in the presence of high concentration of surfactant (10 $\mathrm{mM}$ ), where C-480 molecules are completely solubilized in micelles (excitation wavelength-independent fluorescence peak maximum and very little change in absorption and fluorescence peak maxima in the presence of urea are noted). Figure 4 shows absorption and fluorescence spectra of C-480 in pure water, methanol, cyclohaxane, and $10 \mathrm{mM}$ Gemini-A. Absorption and fluorescence peak maxima of C-480 in pure water are observed at 389 and $489 \mathrm{~nm}$, respectively, and the same in cyclohaxane appeared at 362, 378, and $409 \mathrm{~nm}$, respectively (Table S1). In water, fluorescence of C-480 occurs from the stabilized intramolecular charge transfer (ICT) state. ${ }^{64-67}$ Low fluorescence intensity in a polar medium is due to the high rate of nonradiative processes as triplet and ground states are very close to the ICT state. Compared to water, in a comparatively less polar medium like micelles, fluorescence intensity increases with a concomitant blue shift in peak maximum. This is because the emitting state now gets destabilized and goes away from the triplet and ground states. The blue shift in the absorption peak maximum in water with respect to that in micellar medium is due to the intermolecular hydrogen bonding between C-480 and water molecules, which is generally observed for molecules with

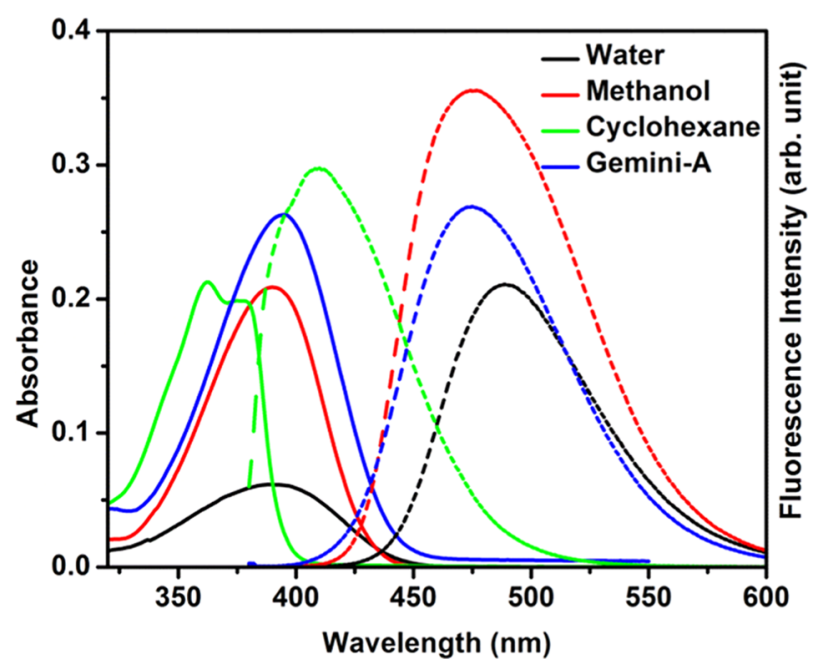

Figure 4. Absorption (solid lines) and fluorescence (dashed lines) spectra $\left(\lambda_{\mathrm{ex}}=375 \mathrm{~nm}\right)$ of C-480 in pure water, methanol, cyclohexane, and $10 \mathrm{mM}$ Gemini-A.

ICT characteristics. ${ }^{64-67}$ The ICT fluorescence of C-480 has also been demonstrated by recording fluorescence spectra in different percentages of dioxane-water mixtures. Figure S1a represents fluorescence spectra and Figure S1b shows changes in fluorescence intensity and fluorescence peak maxima of C-480 with increasing $\%$ of water in dioxane-water mixtures. In the presence of pure gemini surfactants and gemini surfactants plus urea, the absorption and fluorescence peak maxima of C- 480 are different from those in the presence of pure water. These results indicate that the microenvironment around C-480 in the micelles of pure gemini surfactant and that of gemini surfactant plus urea are different from that in the presence of pure water. The blue shift in the fluorescence peak maxima in a micellar medium as well as in a micelle-urea mixed system compared to pure water suggests that C-480 feels less polar environment in micelle and micelle-urea mixed media than that in bulk water.

2.3. Study on Microenvironment of Micellar Systems in the Presence and Absence of Urea. 2.3.1. Micropolarity. Determination of micropolarity around the probe molecule gives valuable information about the location of the probe molecule inside the micellar environment. Fluorescence active probe molecules play an important role in the determination of micropolarity of biological and biologically related environments. $^{50,51}$ In the present study, the micropolarity values expressed in terms of $E_{\mathrm{T}}(30)$, which is an empirical solvent polarity parameter developed by Reichardt et al., ${ }^{68}$ have been estimated to obtain the information about microenvironment surrounding the probe molecule inside the micelles. To determine the micropolarity around C-480 in various studied systems, the fluorescence behaviors of C-480 in the absence and presence of various concentrations of urea have been compared to those in different compositions of dioxane-water mixture. ${ }^{50}$ Fluorescence energies of C-480 at peak maxima $\left(\varepsilon_{\max }\right)$ after correction of $\lambda^{2}$ factor in different percentages of dioxane-water mixture have been plotted against the $E_{\mathrm{T}}(30)$ values of the dioxane-water mixture (Figure S2). The values of $\varepsilon_{\max }{ }^{\mathrm{fl}}$ of C-480 in the absence and presence of various concentrations of urea have been estimated, and the $E_{\mathrm{T}}(30)$ values have been estimated from Figure S2. The $E_{\mathrm{T}}(30)$ values for all of the systems are found to vary between 53.8 and $55.8 \mathrm{kcal} \mathrm{mol}^{-1}$. The calculated $E_{\mathrm{T}}(30)$ values in the investigated systems indicate that 
micropolarity around C-480 in these systems is less than that of water $\left(E_{\mathrm{T}}(30)=63.1 \mathrm{kcal} \mathrm{mol}^{-1}\right)$. The $E_{\mathrm{T}}(30)$ value in all of the studied systems is similar to that of methanol $\left(55.5 \mathrm{kcal} \mathrm{mol}^{-1}\right)$. It depicts that C-480 is present neither at the core of the micelles nor in the bulk water, but somewhere in between these two. According to literature reports, the micropolarity of the Stern layer is similar to methanol, ${ }^{50,51,53,69,70}$ on the basis of which we state that C-480 molecules are mostly residing in the Stern layer. It is pertinent to note that C- 480 being present in the Stern layer of micelles, microenvironment remains more or less same with the addition of urea in micellar systems. Consequently, both fluorescence peak maximum and $E_{\mathrm{T}}(30)$ values are found to be the same in gemini micelles with varying concentrations of urea.

2.3.2. Microviscosity. The measurement of fluorescence anisotropy of a probe molecule in a microenvironment gives information about microviscosity around the molecule. As we did not get a proper trend of microviscosity using C-480 as a probe following our earlier method, ${ }^{50}$ we explored 1,6-diphenyl-1,3,5hexatriene $(\mathrm{DPH})$, which is a well-known viscosity-sensitive fluorescence probe molecule. ${ }^{51}$ The absolute values of microviscosities of micelles, $\eta_{\mathrm{m}}$, using $\mathrm{DPH}$ have been estimated following the Debye-Stokes-Einstein relation (eq 3$)^{51,71}$

$$
\eta_{\mathrm{m}}=k T \tau_{\mathrm{R}} / v_{\mathrm{h}}
$$

where $\tau_{\mathrm{R}}$ is the rotational correlation time of $\mathrm{DPH}, k$ is the Boltzmann constant, $T$ is the kelvin scale temperature, and $v_{\mathrm{h}}$ is the hydrodynamic volume of DPH. The $v_{\mathrm{h}}$ value has been taken as $313 \AA^{3} \cdot{ }^{71}$ The rotational correlation time, $\tau_{\mathrm{R}}$, has been calculated using Perrin's equation (eq 4$)^{72}$

$$
\tau_{\mathrm{R}}=\left\langle\tau_{\mathrm{f}}\right\rangle /\left[\left(r_{\mathrm{o}} / r\right)-1\right]
$$

where $r$ and $\left\langle\tau_{\mathrm{f}}\right\rangle$ are steady-state fluorescence anisotropy and excited-state lifetime of DPH, respectively, and $r_{\mathrm{o}}$ is the steadystate fluorescence anisotropy of DPH in an extremely viscous solvent, whose value is taken as $0.362 .{ }^{73}$ The values of $r,\left\langle\tau_{\mathrm{f}}\right\rangle, \tau_{\mathrm{R}}$ and $\eta_{\mathrm{m}}$ are given in Table 2 . The data in Table 2 show that in case of each micellar system the fluorescence anisotropy and microviscosity decrease with increasing concentration of urea. It further supports our above-mentioned discussion based on $K^{\mathrm{mic}}$ and $\Delta G^{\circ}$ values (Table 1 ) that urea disfavors the formation of micelles. As a result, probe molecules get more and more exposed to the less viscous environment. Although this result does not provide the actual picture of microenvironment around C-480, it serves our purpose to demonstrate the effect of urea concentration on the microviscosity of micelles.

To see whether there is any effect of the nature of spacer group on the microviscosity of micelles, we have plotted $\eta_{\mathrm{m}}$ against varying concentrations of urea for micelles of all three surfactants. Figure 5 shows that at a given concentration of urea microviscosity increases on substitution of the $-\mathrm{OH}$ group in the spacer group and it also increases with increasing number of $-\mathrm{OH}$ groups. This result could be depicting that the hydroxyl group $(s)$ protect $(s)$ the probe molecules from contact with the water molecules due to which the microviscosity of micelles increases. Further evidence in support of this phenomenon has been discussed below.

To further support the effect of urea concentration on the microviscosity of micelles, the excited singlet-state lifetime values of C-480 have been calculated using the time-correlated singlephoton counting (TCSPC) method. Fluorescence decays in all micelle-urea mixed systems are biexponential in nature. The average lifetime has been calculated using eq 5

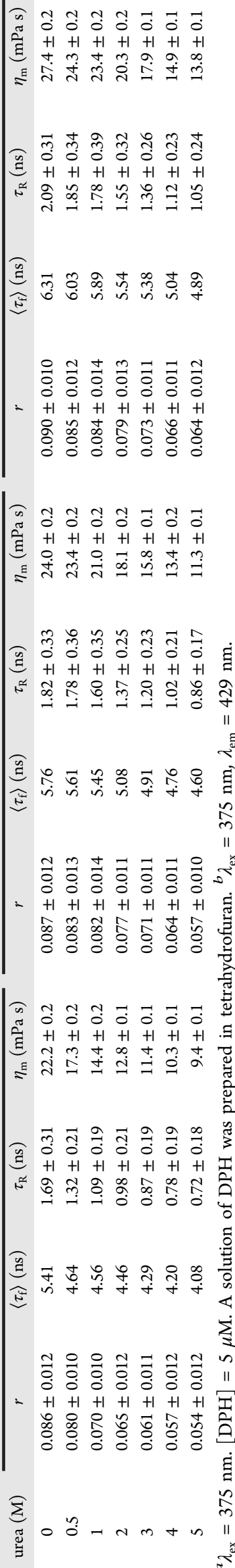




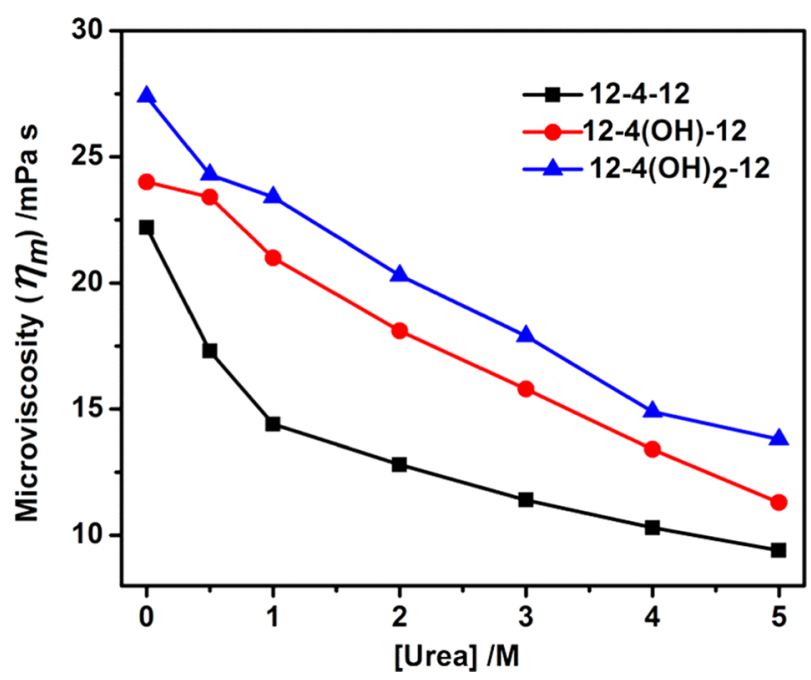

Figure 5. Plot of variation of microviscosity of micelles with increasing concentration of urea in the micellar media of Gemini-A, -B, and -C.

$$
\left\langle\tau_{\mathrm{f}}\right\rangle=a_{1} \tau_{1}+a_{2} \tau_{2}
$$

where $a_{1}$ and $a_{2}$ are the pre-exponential factors for the corresponding lifetimes $\tau_{1}$ and $\tau_{2}$ of the two components. The lifetime values of all components along with the average lifetime of C-480 and $\chi^{2}$ values in all micelle-urea mixed systems are given in Tables S2a-c. The fact that all C-480 molecules are solubilized in micelles at $10 \mathrm{mM}$ concentration of gemini, this biexponential decay could be because of two species: (1) locally excited (LE) and (2) intramolecular charge transfer (ICT) as it is known that C-480 has ICT characteristics. ${ }^{55}$ A detailed study will be carried out later to confirm this. Data in Tables S2a-c show that ICT species is mostly contributing to the decay. It can be seen that average excited-state lifetime values $\left(\left\langle\tau_{\mathrm{f}}\right\rangle\right)$ decreased with increasing urea concentration in all micellar systems. Figure 6 shows the variation of average excited-state lifetime values of $C$ 480 in the presence of all studied surfactants and urea mixed systems. The excited-state lifetime values decreased with increasing microviscosity of gemini surfactants. It has been

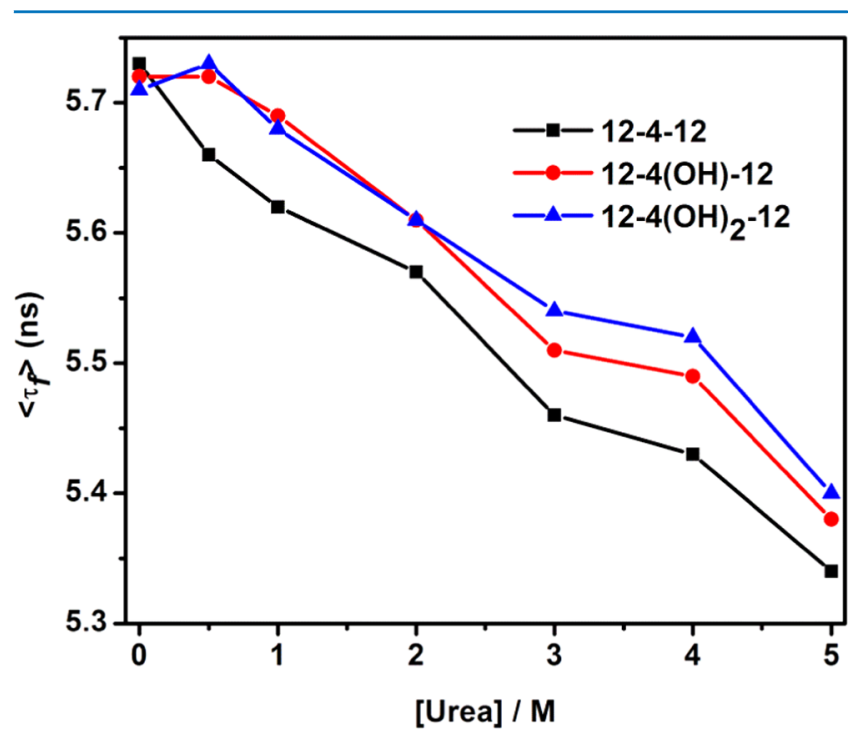

Figure 6. Average excited-state lifetime $\left(\left\langle\tau_{\mathrm{f}}\right\rangle\right)$ value of C-480 in the presence of the studied mixed systems. [Surfactant] $=10 \mathrm{mM}$. $\lambda_{\mathrm{ex}}=375$ $\mathrm{nm}, \lambda_{\mathrm{em}}=475 \mathrm{~nm}$. mentioned (Table S1) that the fluorescence peak maxima of $\mathrm{C}$ 480 remains unchanged in the presence and absence of urea. It infers that variation in excited singlet-state lifetime is mostly due to change in microviscosity of the systems. Of course, a comparatively less effect due to the change in micropolarity cannot be ruled out.

2.4. Solvation Dynamics. Solvation dynamics of C-480 has been studied in the presence of $10 \mathrm{mM}$ concentration of all three studied gemini surfactants and in the presence of gemini surfactant with various concentrations of urea in the range mentioned above. Fluorescence decays of C-480 in $10 \mathrm{mM}$ concentration of all of the three gemini surfactants with varying concentrations of urea have been recorded. In these systems, emission wavelength-dependent decays of C-480 have been noted. Wavelengths are selected from the entire range of a steady-state fluorescence spectrum of C-480 (Figure 3). Figure 7

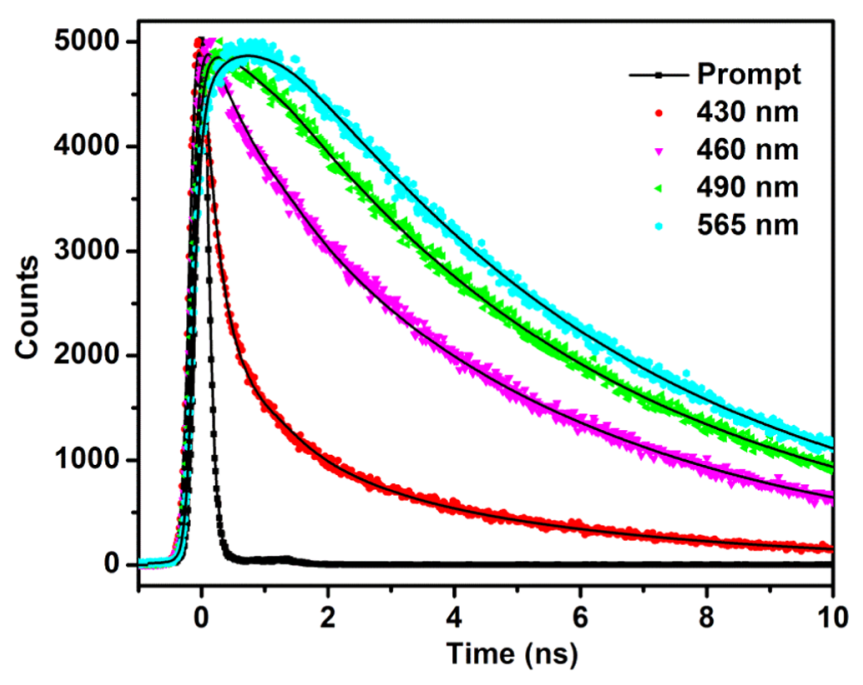

Figure 7. Fluorescence decays of C-480 in $12-4-12(10 \mathrm{mM})$ at $5 \mathrm{M}$ urea concentration. $\lambda_{\mathrm{ex}}=375 \mathrm{~nm}$.

shows the emission wavelength-dependent decays of C-480 in $12-4-12(10 \mathrm{mM})$ at $5 \mathrm{M}$ urea concentration as a representative one. A fast decay is noted at a short wavelength (say $430 \mathrm{~nm}$ ). Fluorescence occurring from the unsolvated dipole generated at the excited state is responsible for fast decay. Of course, there is a possibility of contribution of high percentage of solvated dipoles to the fast decay, which could not be detected due to the shortcomings of our TCSPC setup (instrument response function $=165 \mathrm{ps}$ ). At a longer wavelength (say $565 \mathrm{~nm}$ ), fluorescence decay shows a clear growth in the spectrum, followed by the decay. The growth in the decay indicates the solvation of the probe molecule in the exited state. ${ }^{74}$ Decays were fitted triexponentially at a shorter wavelength, whereas biexponentially at a longer wavelength. Figure S3a,b shows decays of C-480 in $12-4(\mathrm{OH})-12(10 \mathrm{mM})$ at $5 \mathrm{M}$ urea concentration and $12-4(\mathrm{OH})_{2}-12(10 \mathrm{mM})$ at $5 \mathrm{M}$ urea concentration as representatives. Similar behaviors have also been observed for all gemini surfactants and urea mixed systems. It is mentioned above that the most probable location of C-480 molecules is the Stern layer. Therefore, the probe molecules located at the Stern layer of the micelles are mostly contributing to the said solvation process. ${ }^{50,51,75}$ The solvation processes occurring in the bulk are too fast to be measured by our instrumental setup (time resolution, $\sim 165 \mathrm{ps}$ ). On the other hand, the probe molecules located in the hydrocarbon core of the 

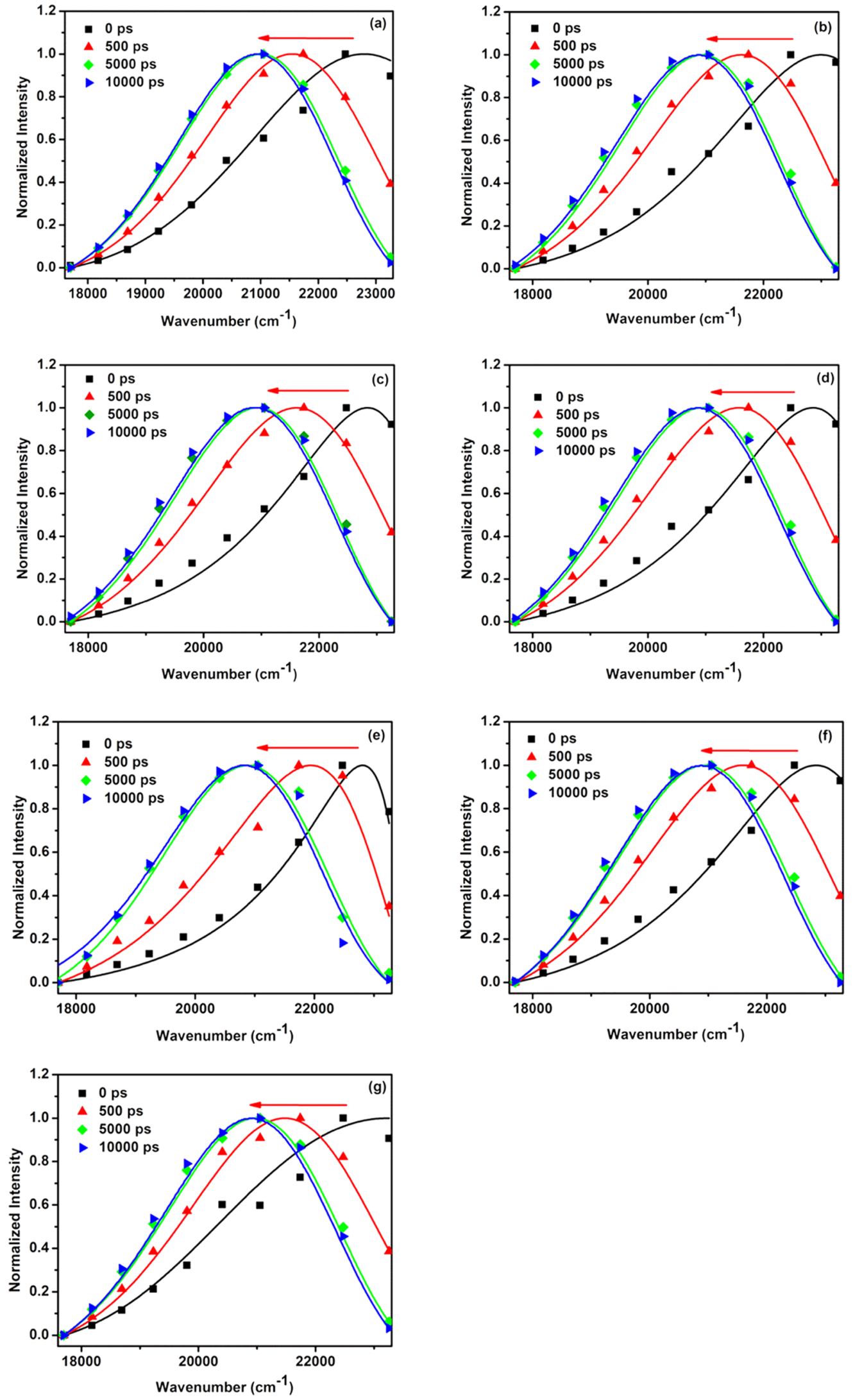

Figure 8. Time-resolved emission spectra of C-480 in 12-4-12 (10 mM) at $X \mathrm{M}$ urea (from right to left: 0, 500, 5000, and $10000 \mathrm{ps}$ ): $X=0 \mathrm{M}$ (a), $0.5 \mathrm{M}$ (b), 1.0 M (c), 2.0 M (d), 3.0 M (e), 4.0 M (f), and 5.0 M (g).

micelles are not supposed to contribute to any solvation process. $^{50,51}$
Time-resolved emission spectra (TRES) have been constructed by following the method of Fleming and Maroncelli. ${ }^{76}$ 

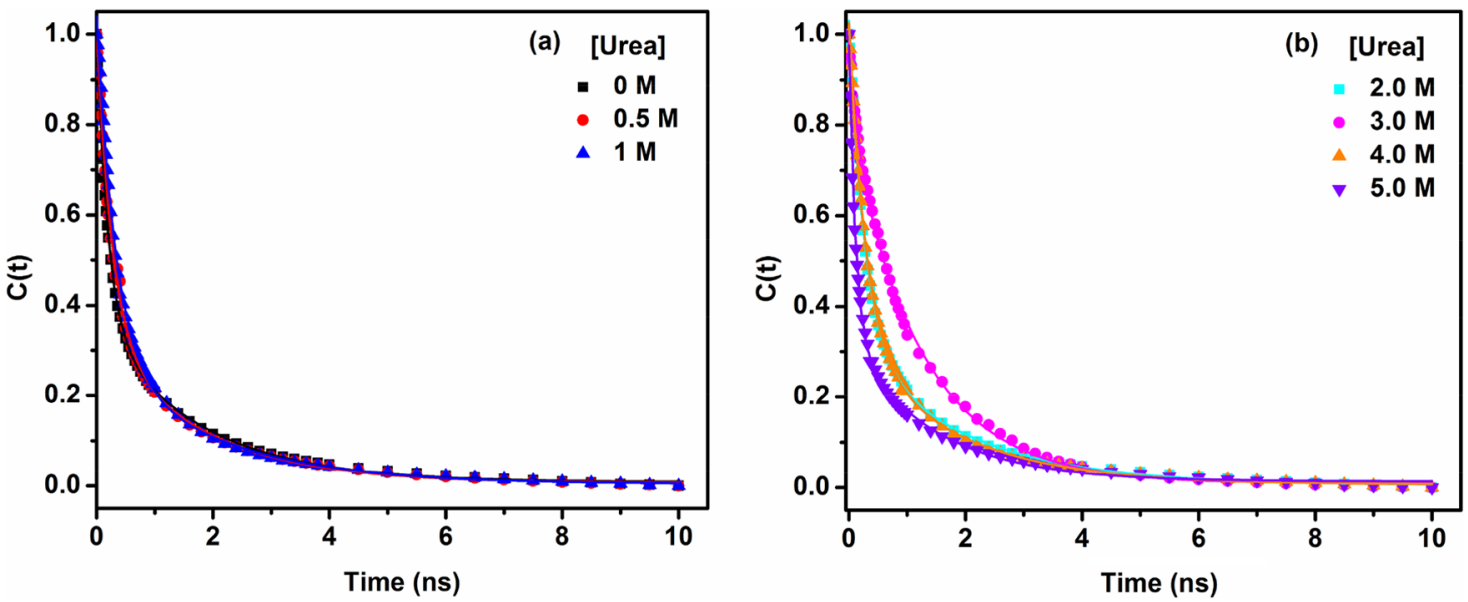

Figure 9. Decays of solvent response function, $C(t)$, of C-480 in the micelles of Gemini-A and urea. (a) 0-1 M urea and (b) 2-5 M urea.

Table 3a. Decay Characteristic of Solvent Response Function, $C(t)$, of C-480 in the Presence of Pure 12-4-12 and 12-4-12 at Various Concentrations of Urea ${ }^{a}$

\begin{tabular}{ccccccc} 
urea $(\mathrm{M})$ & $a_{1 \mathrm{~s}}$ & $\tau_{1 \mathrm{~s}}(\mathrm{ps})$ & $a_{2 \mathrm{~s}}$ & $\tau_{2 \mathrm{~s}}(\mathrm{ps})$ & $\left\langle\tau_{\mathrm{s}}\right\rangle(\mathrm{ps})$ & $\Delta \nu\left(\mathrm{cm}^{-1}\right)$ \\
0.0 & $0.64 \pm 0.01$ & $193.15 \pm 0.01$ & $0.36 \pm 0.01$ & $1709.25 \pm 0.04$ & 738.95 & 1855 \\
0.5 & $0.66 \pm 0.02$ & $258.12 \pm 0.01$ & $0.34 \pm 0.02$ & $1706.93 \pm 0.12$ & 750.72 & 1917 \\
1.0 & $0.73 \pm 0.02$ & $321.99 \pm 0.01$ & $0.27 \pm 0.02$ & $1986.20 \pm 0.21$ & 771.33 & 1956 \\
2.0 & $0.69 \pm 0.01$ & $270.65 \pm 0.01$ & $0.31 \pm 0.01$ & $1919.62 \pm 0.11$ & 781.83 & 1995 \\
3.0 & $0.69 \pm 0.01$ & $373.04 \pm 0.01$ & $0.31 \pm 0.01$ & $1869.15 \pm 0.08$ & 836.83 & 1989 \\
4.0 & $0.71 \pm 0.01$ & $285.66 \pm 0.01$ & $0.29 \pm 0.01$ & $1778.84 \pm 0.10$ & 718.68 & 1957 \\
5.0 & $0.67 \pm 0.01$ & $115.14 \pm 0.01$ & $0.33 \pm 0.01$ & $1431.39 \pm 0.07$ & 549.50 & 2314 \\
${ }^{a} \Delta \nu=\nu(0)-\nu(\infty)$. & & & & & & \\
\hline
\end{tabular}

Table 3b. Decay Characteristic of Solvent Response Function, $C(t)$, of C-480 in the Presence of Pure 12-4(OH)-12 and 12-4(OH)12 at Various Concentrations of Urea

\begin{tabular}{ccccccc}
\hline urea $(\mathrm{M})$ & $a_{1 \mathrm{~s}}$ & $\tau_{\mathrm{ss}}(\mathrm{ps})$ & $a_{2 \mathrm{~s}}$ & $\tau_{2 \mathrm{~s}}(\mathrm{ps})$ & $\left\langle\tau_{\mathrm{s}}\right\rangle(\mathrm{ps})$ & $\Delta \nu\left(\mathrm{cm}^{-1}\right)$ \\
\hline 0.0 & $0.68 \pm 0.01$ & $355.43 \pm 0.01$ & $0.28 \pm 0.01$ & $1861.01 \pm 0.08$ & 762.76 & 1910 \\
0.5 & $0.56 \pm 0.04$ & $297.45 \pm 0.01$ & $0.44 \pm 0.04$ & $1409.21 \pm 0.25$ & 786.62 & 1930 \\
1.0 & $0.66 \pm 0.02$ & $347.02 \pm 0.01$ & $0.34 \pm 0.02$ & $1735.24 \pm 0.17$ & 819.01 & 2057 \\
2.0 & $0.52 \pm 0.03$ & $337.47 \pm 0.02$ & $0.48 \pm 0.03$ & $1498.59 \pm 0.13$ & 894.81 & 1954 \\
3.0 & $0.73 \pm 0.02$ & $371.56 \pm 0.01$ & $0.27 \pm 0.02$ & $1768.15 \pm 0.16$ & 748.64 & 1911 \\
4.0 & $0.51 \pm 0.01$ & $262.44 \pm 0.01$ & $0.49 \pm 0.01$ & $1230.69 \pm 0.02$ & 736.88 & 2098 \\
5.0 & $0.67 \pm 0.01$ & $175.85 \pm 0.01$ & $0.33 \pm 0.01$ & $1350.64 \pm 0.03$ & 563.53 & 2366 \\
${ }^{a} \Delta \nu=\nu(0)-\nu(\infty)$. & & & & & \\
\hline
\end{tabular}

Table 3c. Decay Characteristic of Solvent Response Function, $C(t)$, of C-480 in the Presence of Pure 12-4(OH $)_{2}-12$ and 12 $4(\mathrm{OH})_{2}-12$ at Various Concentrations of Urea ${ }^{a}$

\begin{tabular}{ccccccc} 
urea $(\mathrm{M})$ & $a_{1 \mathrm{~s}}$ & $\tau_{1 \mathrm{~s}}(\mathrm{ps})$ & $a_{2 \mathrm{~s}}$ & $\tau_{2 \mathrm{~s}}(\mathrm{ps})$ & $\left\langle\tau_{\mathrm{s}}\right\rangle(\mathrm{ps})$ & $\Delta \nu\left(\mathrm{cm}^{-1}\right)$ \\
0.0 & $0.80 \pm 0.04$ & $493.77 \pm 0.02$ & $0.20 \pm 0.03$ & $2589.21 \pm 0.61$ & 912.86 & 2075 \\
0.5 & $0.82 \pm 0.03$ & $521.95 \pm 0.02$ & $0.18 \pm 0.03$ & $2847.76 \pm 0.69$ & 940.60 & 2050 \\
1.0 & $0.82 \pm 0.04$ & $535.06 \pm 0.02$ & $0.18 \pm 0.03$ & $2930.32 \pm 0.71$ & 966.21 & 2030 \\
2.0 & $0.86 \pm 0.03$ & $515.85 \pm 0.03$ & $0.14 \pm 0.02$ & $4721.38 \pm 0.73$ & 1104.62 & 1997 \\
3.0 & $0.70 \pm 0.02$ & $391.78 \pm 0.01$ & $0.30 \pm 0.02$ & $1743.48 \pm 0.11$ & 797.29 & 1930 \\
4.0 & $0.68 \pm 0.02$ & $392.63 \pm 0.01$ & $0.32 \pm 0.02$ & $1580.51 \pm 0.07$ & 772.75 & 1885 \\
5.0 & $0.75 \pm 0.02$ & $376.72 \pm 0.01$ & $0.25 \pm 0.02$ & $1867.86 \pm 0.13$ & 749.51 & 1820 \\
${ }^{a} \Delta \nu=\nu(0)-\nu(\infty)$. & & & & & & \\
\hline
\end{tabular}

Dynamic Stokes shifts in the emission spectra of C-480 can be seen after constructing TRES. Figure 8 shows the TRES of C-480 in the presence of $12-4-12(10 \mathrm{mM})$ at varying concentrations of urea as a representative one. TRES for other systems are given in the Supporting Information (Figure S4a,b). The peak wavenumber, $v(t)$, for all TRES at different times was obtained after fitting the TRES to a log-normal function. ${ }^{66,76}$ The peak wavenumber $(v(t))$ values have been used to calculate the solvent response function (SRF), $C(t)$, using eq 6 given by Fleming and Maroncelli ${ }^{76}$ for the quantitative measurement of solvation dynamics 


$$
C(t)=\frac{v(t)-v(\infty)}{v(0)-v(\infty)}
$$

where $v(0), v(t)$, and $v(\infty)$ are the peak wavenumbers at times zero, $t$, and infinity, respectively. Figure 9 shows the plot of $C(t)$ versus time for the system of $12-4-12(10 \mathrm{mM})$ at varying concentration of urea. The $C(t)$ decays for other systems are given in the Supporting Information (Figure S5a,b). The $C(t)$ decays are generally biexponential in nature in all of the studied systems. The biexponential fitting of decays of $C(t)$ has been done using eq 7

$$
C(t)=a_{1 \mathrm{~s}} \mathrm{e}^{-t / \tau_{1 \mathrm{~s}}}+a_{2 \mathrm{~s}} \mathrm{e}^{-t / \tau_{2 \mathrm{~s}}}
$$

where $\tau_{1 \mathrm{~s}}$ and $\tau_{2 \mathrm{~s}}$ represent the solvent relaxation times, and $a_{1 \mathrm{~s}}$ and $a_{2 s}$ are the corresponding amplitudes. The decay characteristics of $C(t)$ are given in Tables $3 a-3 c$ for all of the studied systems. The average solvation time, $\left\langle\tau_{\mathrm{s}}\right\rangle$, for a biexponential decay has been estimated by eq 8 and are also given in Tables $3 a-3 c$

$$
\left\langle\tau_{\mathrm{s}}\right\rangle=a_{1 \mathrm{~s}} \tau_{1 \mathrm{~s}}+a_{2 \mathrm{~s}} \tau_{2 \mathrm{~s}}
$$

As mentioned in the literature, the bimodal behavior of solvation in the microheterogeneous systems arises due to the presence of free and bound water molecules. ${ }^{77}$ The strength of hydrogen bond between the water molecule and polar headgroup is stronger than that between two water molecules. ${ }^{70,78,79}$ The water molecules that are hydrogen-bonded with the polar headgroups are called bound water. Free water molecules lead to fast solvation, whereas the bound water molecules are responsible for slow solvation. ${ }^{80}$ In all micellar systems, the fast components are the major components to contribute to the solvation processes. In case of gemini surfactant systems, polar headgroups, counterions, and spacer group can also contribute to the solvation process. For a gemini surfactant, the polar headgroups are joined to the long hydrocarbon tails as well as to the spacer part. The mobilities of polar headgroups as well as spacer group are restricted greatly as they are connected directly/ indirectly to the long hydrocarbon tails, and it is well reported that polymer chain dynamics takes place on a very slow time scale $(\sim 100 \mathrm{~ns}) .^{50-53,70,75}$ Therefore, essentially counterions and water molecules are responsible for the solvation processes.

The values of average solvation time $\left(\left\langle\tau_{s}\right\rangle\right)$ of C-480 in the presence of gemini surfactants and urea mixed systems have been calculated (Tables $3 a-3 c$ ) using eq 8 , and their variations with urea concentration are shown in Figure 10. It can be observed from the data in Tables $3 a-3 c$ as well as from Figure 10 that in the absence of urea and also up to $2.0 \mathrm{M}$ concentration of urea, the average solvation time increases with increasing number of hydroxyl groups in the spacer group of gemini surfactants. ${ }^{50}$ As reported by us earlier, ${ }^{50}$ the hydroxyl group $(s)$ present in the spacer group of gemini surfactant might be forming hydrogen bonds with the water molecules. It protects the probe molecules from contact with the water molecules and that is why solvation time is increased with increasing number of hydroxyl groups in the spacer group of gemini surfactants. ${ }^{50}$ Recently, we have reported comparatively slower solvation processes in case of micelles of gemini surfactants containing diethyl ether spacer group causing similar effect. ${ }^{74}$ In case of micelles of Gemini-A, i.e., 12-4-12 surfactant, because of the presence of hydrophobic spacer group, the extent of free water molecules is greater than that in the micelles of gemini surfactants possessing hydroxyl groups in the spacer group. Evidence to this hypothesis has been discussed below.

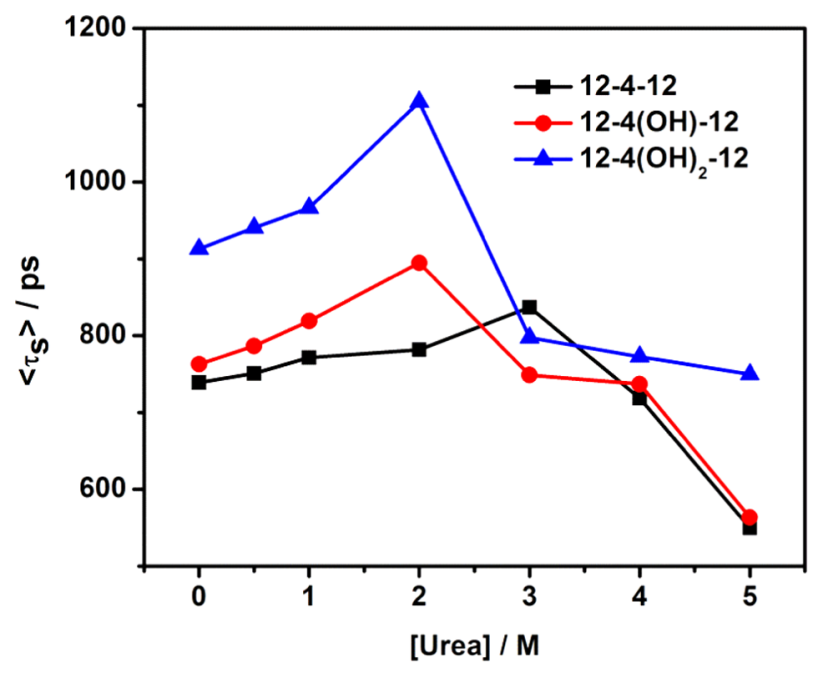

Figure 10. Average solvation time of C-480 in the presence of gemini surfactant and urea mixed systems.

We have carried out a separate fluorescence experiment to further support the fact that the hydroxyl group(s) present in the spacer group of gemini surfactants protects the probe molecules from contact with a certain amount of water molecules. ${ }^{74}$ It has been reported ${ }^{61,81}$ that the significant change in micelle size and $\mathrm{cmc}$ occurs only when the percentage of organic co-solvent in water-organic solvent mixture is above $20 \%$. In view of this fact, we have recorded fluorescence spectra of C-480 in aqueous micelles and also in micelles in the presence of various percentages of organic co-solvent not exceeding 20\%. By performing these experiments, we wanted to demonstrate the extent of interactions between the hydroxyl group of methanol and C-480. For this purpose, we have presented here Figure 11a for Gemini-A, which has been reported earlier. ${ }^{74}$ This figure shows that in the presence of $10 \mathrm{mM}$ Gemini-A the fluorescence peak maximum gets red-shifted from 477 to $480 \mathrm{~nm}$ on increasing the percentage of methanol up to $20 \%$. However, Figure $11 \mathrm{~b}, \mathrm{c}$ shows that there is no such change in fluorescence peak maximum in $10 \mathrm{mM}$ Gemini-B and Gemini-C, respectively, in the presence of the same percentage of methanol. Thus, these results depict that hydroxyl group(s) present in the spacer group of Gemini-B and Gemini-C protect molecules containing - $\mathrm{OH}$ groups from contact with probe molecule. Also this experiment is an extra step compared to our previous study ${ }^{50}$ to support our hypothesis of protection of water molecules by $-\mathrm{OH}$ group(s) in the spacer groups.

One can see in Figure 10 that with increasing concentration of urea the average solvation time initially increases, reaches a maximum, and then decreases for all three gemini micellar systems. For Gemini-B and Gemini-C, the solvation time is maximum at $2.0 \mathrm{M}$ of urea, whereas for Gemini-A, the solvation time is maximum at $3.0 \mathrm{M}$ of urea, which can be due to the fact that the concentration of urea has an effect on the degree of counterion dissociation $(\alpha)$ (Table 4 ) as well.

Figure 12 represents the variation of $\alpha$ with increasing concentration of urea, that is, $\alpha$ increases, reaches a maximum, and then decreases with increasing concentration of urea. The concentration of urea at which $\alpha$ is maximum is the same as that of average solvation time. There is a good correlation between the variation of average solvation time and $\alpha$ with increasing concentration of urea in each case of gemini surfactant. These results show that counterions have an impact on the solvation 

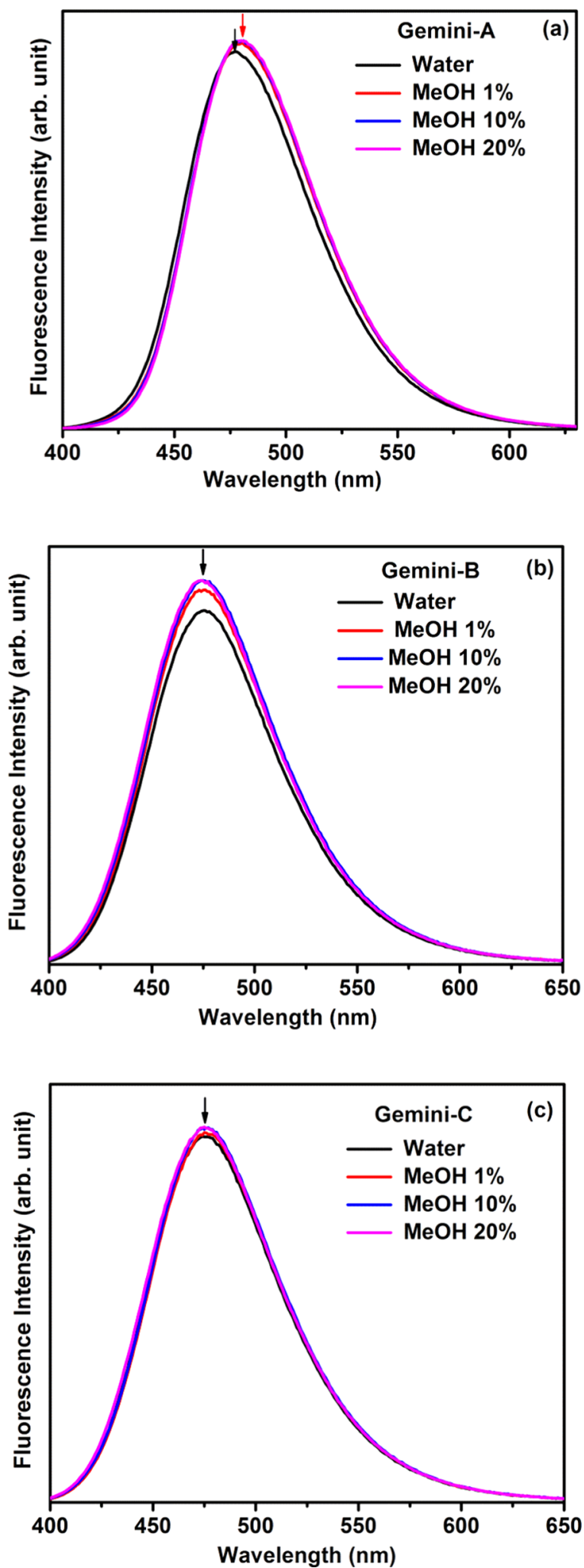

Figure 11. Fluorescence spectra of C-480 in $10 \mathrm{mM}$ (a) Gemini-A, (b) Gemini-B, and (c) Gemini-C in the presence of various percentages of methanol in water-methanol mixture. $\lambda_{\mathrm{ex}}=375 \mathrm{~nm}$.

dynamics. In our previous study with gemini surfactants with polymethylene spacer group and also with mixed surfactant systems, we have found that the counterions have an impact on solvation dynamics. ${ }^{51,53}$ With increasing $\alpha$, the extent of free counterions increases. It is known that water molecules can form
Table 4. Degree of Counterion Dissociation ( $\alpha$ ) for Micelles of Gemini Surfactants

$\begin{array}{cccc}\text { urea }(\mathrm{M}) & \text { Gemini-A } \alpha & \text { Gemini-B } \alpha & \text { Gemini-C } \alpha \\ 0.0 & 0.32 \pm 0.03 & 0.29 \pm 0.02 & 0.28 \pm 0.02 \\ 0.5 & 0.36 \pm 0.02 & 0.37 \pm 0.03 & 0.29 \pm 0.02 \\ 1.0 & 0.42 \pm 0.04 & 0.38 \pm 0.03 & 0.32 \pm 0.02 \\ 2.0 & 0.46 \pm 0.04 & 0.39 \pm 0.03 & 0.37 \pm 0.03 \\ 3.0 & 0.47 \pm 0.04 & 0.36 \pm 0.02 & 0.34 \pm 0.02 \\ 4.0 & 0.34 \pm 0.02 & 0.33 \pm 0.02 & 0.32 \pm 0.02 \\ 5.0 & 0.31 \pm 0.02 & 0.30 \pm 0.02 & 0.27 \pm 0.02\end{array}$

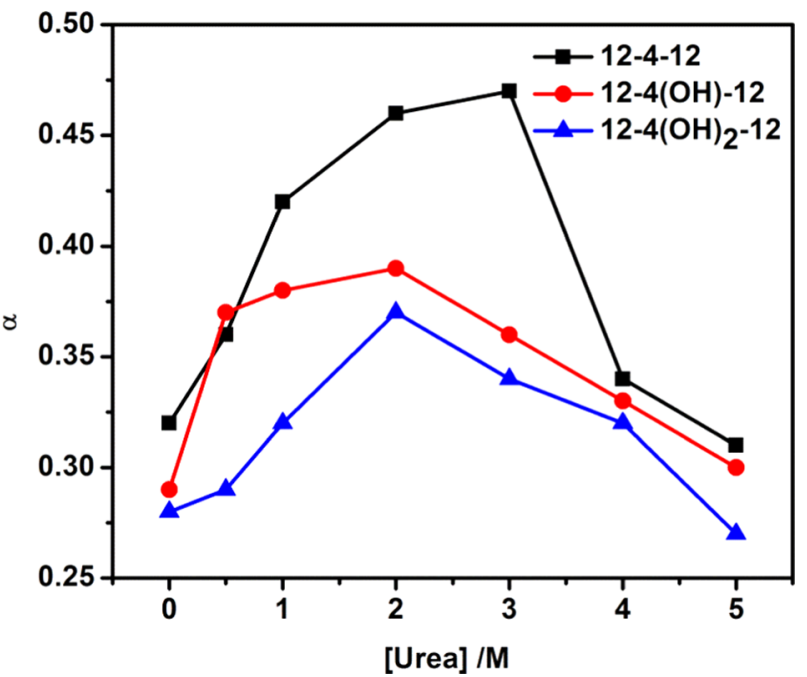

Figure 12. Degree of counterion dissociation $(\alpha)$ vs concentration of urea for the micelles of Gemini-A, Gemini-B, and Gemini-C.

hydrogen bonds with anions. ${ }^{82}$ Thus, up to a certain concentration of urea with increasing number of counterions, the clustering of water molecules increases. ${ }^{83}$ With a greater degree of clustering of water molecules, the number of free water molecules is expected to decrease. This could be the reason for the solvation process becoming slower. However, above a certain concentration of urea with decreasing $\alpha$ (decreasing number of free counterions), the clustering of water molecules is reduced. Because of this, the number of free water molecules probably starts increasing, which results in an increase in the rate of solvation process, i.e., the solvation time starts decreasing. Thus, free counterions indirectly control the solvation dynamics via the formation of water clusters. It is noteworthy that the ascending order of $\alpha$ observed in the micelles of surfactants at a given concentration of urea is as follows: Gemini-C $<$ Gemini-B < Gemini-A. However, the order is reversed as far as the average solvation times are concerned at a given concentration of urea. It infers that ultimately the net availability of free water molecules toward the solvation process of solute molecules probably controls the rate of solvation. Although the clustering of water molecules is higher in case of Gemini-A due to comparatively greater $\alpha$, because of the presence of hydrophobic spacer group, the accessibility of free water molecules for effective solvation might be larger in this case. Thus, spacer group of gemini surfactant plays an important role in solvation dynamics. It is to be mentioned here that even high concentration of urea has very less effect on the strength of hydrogen-bonding interactions between water molecules. ${ }^{38}$ Only a small fraction of water molecules gets immobilized by urea. Therefore, any significant direct effect of urea on the solvation dynamics can be ruled out. 

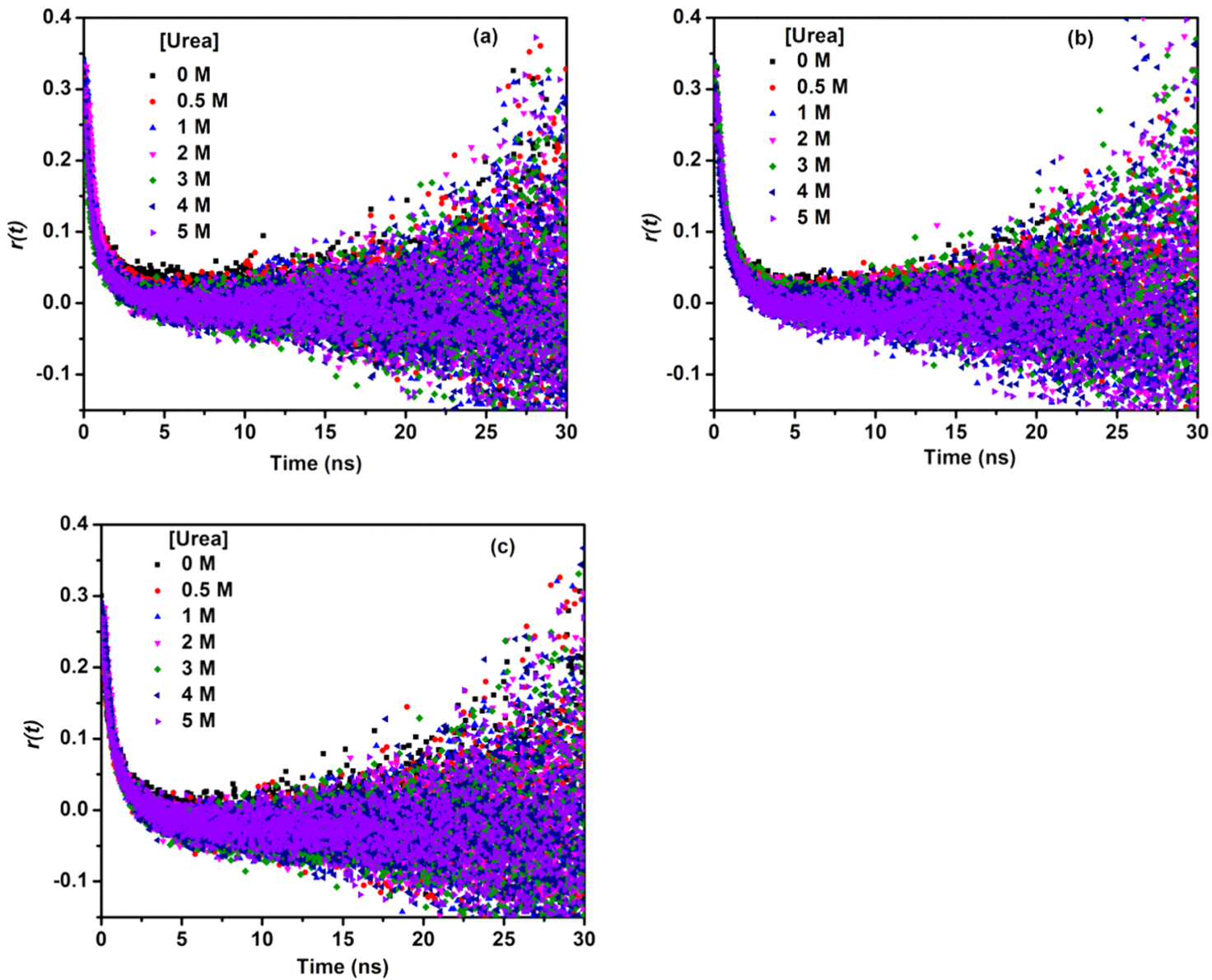

Figure 13. Fluorescence anisotropy decays of C-480 in the micelles of gemini surfactants: (a) Gemini-A, (b) Gemini-B, and (c) Gemini-C in the presence of various concentrations of urea. $\lambda_{\mathrm{ex}}=375 \mathrm{~nm}$. $\lambda_{\mathrm{em}}=470 \mathrm{~nm}$.

Table 5a. Rotational Relaxation Parameters of C-480 in the Micelles of Gemini-A and Urea Mixed Systems ${ }^{a}$

\begin{tabular}{ccccccc} 
urea $(\mathrm{M})$ & $r_{0}$ & $a_{1 \mathrm{r}}$ & $\tau_{1 \mathrm{r}}(\mathrm{ns})$ & $a_{2 \mathrm{r}}$ & $\tau_{2 \mathrm{r}}(\mathrm{ns})$ & $\left\langle\tau_{\mathrm{r}}\right\rangle(\mathrm{ns})$ \\
0.0 & $0.34 \pm 0.04$ & $0.87 \pm 0.02$ & $0.61 \pm 0.06$ & $0.13 \pm 0.02$ & $6.91 \pm 0.53$ & 1.43 \\
0.5 & $0.33 \pm 0.05$ & $0.90 \pm 0.02$ & $0.67 \pm 0.07$ & $0.10 \pm 0.02$ & $7.41 \pm 0.61$ & 1.34 \\
1.0 & $0.31 \pm 0.03$ & $0.92 \pm 0.01$ & $0.66 \pm 0.06$ & $0.08 \pm 0.01$ & $7.74 \pm 0.63$ & 1.23 \\
2.0 & $0.29 \pm 0.03$ & $0.93 \pm 0.01$ & $0.67 \pm 0.07$ & $0.07 \pm 0.01$ & $6.96 \pm 0.56$ & 1.11 \\
3.0 & $0.28 \pm 0.04$ & $0.62 \pm 0.02$ & $0.36 \pm 0.04$ & $0.38 \pm 0.01$ & $1.73 \pm 0.12$ & 0.88 \\
4.0 & $0.27 \pm 0.03$ & $0.74 \pm 0.02$ & $0.45 \pm 0.05$ & $0.26 \pm 0.01$ & $2.09 \pm 0.14$ & 0.88 \\
$a \lambda_{\text {ex }}=375 \mathrm{~nm}, \lambda_{\mathrm{em}}=$ & $0.27 \pm 0.03$ & $0.70 \pm 0.02$ & $0.39 \pm 0.04$ & $0.30 \pm 0.01$ & $1.96 \pm 0.13$ & 0.86 \\
\hline
\end{tabular}

2.5. Time-Resolved Fluorescence Anisotropy. To obtain further information about the microenvironment of micelles, the time-resolved fluorescence anisotropy, $r(t)$, measurement in molecular assemblies has been carried out. ${ }^{51-53}$ The $r(t)$ values have been determined using eq 9

$$
r(t)=\frac{I_{\|}(t)-G I_{\perp}(t)}{I_{\|}(t)+2 G I_{\perp}(t)}
$$

where $I_{\perp}(t)$ and $I_{\|}(t)$ are the fluorescence decays polarized perpendicular and parallel to the polarization of the excitation light, respectively. $G$ in eq 9 is the correction factor for detector sensitivity to the polarization detection of emission. For our instrument setup, $G$ factor is $\sim 0.6$. Rotational relaxation behaviors of C-480 in micelles of gemini surfactants and in the presence of various concentrations of urea have been characterized. Figure $13 a-c$ shows the fluorescence anisotropy decays of C-480 in Gemini-A, $-\mathrm{B}$, and $-\mathrm{C}$, respectively, in the presence of various concentrations of urea. In pure water, the anisotropy decay of C-480 is monoexponential, whereas that in the presence of pure gemini surfactants and also in the presence of gemini surfactant with various concentrations of added urea is biexponential in nature. The decay function represented by eq 10 has been applied to fit the biexponential anisotropy decay

$$
r(t)=r_{0}\left[a_{1 \mathrm{r}} \mathrm{e}^{-t / \tau_{1 \mathrm{r}}}+a_{2 \mathrm{r}} \mathrm{e}^{-t / \tau_{2 \mathrm{r}}}\right]
$$

where $r_{0}$ is the limiting value of anisotropy, which represents the inherent depolarization of the probe molecule, $\tau_{1 \mathrm{r}}$ and $\tau_{2 r}$ represent the time constants for the fast and slow rotational relaxation components, respectively, and $a_{1 \mathrm{r}}$ and $a_{2 \mathrm{r}}$ are the corresponding relative amplitudes. The rotational relaxation times for the fast and slow components have been obtained from 
Table 5b. Rotational Relaxation Parameters of C-480 in the Micelles of Gemini-B and Urea Mixed Systems ${ }^{a}$

\begin{tabular}{|c|c|c|c|c|c|c|}
\hline urea (M) & $r_{0}$ & $a_{1 \mathrm{r}}$ & $\tau_{\mathrm{lr}}(\mathrm{ns})$ & $a_{2 \mathrm{r}}$ & $\tau_{2 \mathrm{r}}(\mathrm{ns})$ & $\left\langle\tau_{\mathrm{r}}\right\rangle(\mathrm{ns})$ \\
\hline 0.0 & $0.33 \pm 0.05$ & $0.91 \pm 0.02$ & $0.67 \pm 0.07$ & $0.09 \pm 0.01$ & $13.13 \pm 0.98$ & 1.79 \\
\hline 0.5 & $0.32 \pm 0.04$ & $0.93 \pm 0.02$ & $0.69 \pm 0.07$ & $0.07 \pm 0.01$ & $10.39 \pm 0.87$ & 1.37 \\
\hline 1.0 & $0.33 \pm 0.05$ & $0.91 \pm 0.01$ & $0.63 \pm 0.06$ & $0.09 \pm 0.01$ & $7.97 \pm 0.65$ & 1.29 \\
\hline 2.0 & $0.32 \pm 0.03$ & $0.89 \pm 0.01$ & $0.62 \pm 0.05$ & $0.11 \pm 0.01$ & $5.29 \pm 0.44$ & 1.13 \\
\hline 3.0 & $0.31 \pm 0.03$ & $0.90 \pm 0.01$ & $0.65 \pm 0.06$ & $0.10 \pm 0.01$ & $5.09 \pm 0.43$ & 1.09 \\
\hline 4.0 & $0.30 \pm 0.03$ & $0.78 \pm 0.01$ & $0.49 \pm 0.05$ & $0.22 \pm 0.01$ & $2.51 \pm 0.15$ & 0.93 \\
\hline 5.0 & $0.27 \pm 0.03$ & $0.78 \pm 0.01$ & $0.52 \pm 0.05$ & $0.22 \pm 0.01$ & $2.25 \pm 0.14$ & 0.90 \\
\hline
\end{tabular}

Table 5c. Rotational Relaxation Parameters of C-480 in the Micelles of Gemini-C and Urea Mixed Systems ${ }^{a}$

\begin{tabular}{|c|c|c|c|c|c|c|}
\hline urea $(\mathrm{M})$ & $r_{0}$ & $a_{1 \mathrm{r}}$ & $\tau_{1 \mathrm{r}}(\mathrm{ns})$ & $a_{2 \mathrm{r}}$ & $\tau_{2 \mathrm{r}}(\mathrm{ns})$ & $\left\langle\tau_{\mathrm{r}}\right\rangle(\mathrm{ns})$ \\
\hline 0.0 & $0.30 \pm 0.04$ & $0.85 \pm 0.01$ & $0.76 \pm 0.08$ & $0.15 \pm 0.02$ & $12.82 \pm 0.97$ & 2.57 \\
\hline 0.5 & $0.28 \pm 0.03$ & $0.86 \pm 0.02$ & $0.75 \pm 0.08$ & $0.14 \pm 0.01$ & $12.38 \pm 0.89$ & 2.38 \\
\hline 1.0 & $0.28 \pm 0.03$ & $0.90 \pm 0.02$ & $0.79 \pm 0.08$ & $0.10 \pm 0.01$ & $12.68 \pm 0.98$ & 1.98 \\
\hline 2.0 & $0.27 \pm 0.03$ & $0.91 \pm 0.01$ & $0.78 \pm 0.08$ & $0.09 \pm 0.01$ & $13.25 \pm 0.99$ & 1.90 \\
\hline 3.0 & $0.27 \pm 0.03$ & $0.87 \pm 0.02$ & $0.73 \pm 0.07$ & $0.13 \pm 0.01$ & $7.19 \pm 0.59$ & 1.57 \\
\hline 4.0 & $0.26 \pm 0.03$ & $0.89 \pm 0.02$ & $0.72 \pm 0.07$ & $0.11 \pm 0.01$ & $7.84 \pm 0.63$ & 1.50 \\
\hline 5.0 & $0.26 \pm 0.03$ & $0.57 \pm 0.02$ & $0.39 \pm 0.05$ & $0.43 \pm 0.02$ & $1.89 \pm 0.12$ & 1.04 \\
\hline
\end{tabular}

the fitted decays. The average rotational relaxation time has been determined using eq 11

$$
\left\langle\tau_{\mathrm{r}}\right\rangle=a_{1 \mathrm{r}} \tau_{1 \mathrm{r}}+a_{2 \mathrm{r}} \tau_{2 \mathrm{r}}
$$

where $\tau_{1 \mathrm{r}}$ and $\tau_{2 \mathrm{r}}$ are the rotational relaxation times, and $a_{1 \mathrm{r}}$ and $a_{2 \mathrm{r}}$ are the corresponding amplitudes.

Tables $5 a-5 c$ present the rotational relaxation parameters along with the average rotational relaxation times of $\mathrm{C}-480$ in the micelles of Gemini-A, -B, and -C, respectively, in the presence of various concentrations of urea. The rotational relaxation time of C-480 in the presence of pure water is found to be $132 \mathrm{ps,} \mathrm{which}$ is in good agreement with the reported value of $125 \mathrm{ps}{ }^{84}$ The rotational relaxation time of C-480 in micelles is many fold slower compared to that in pure water. This suggests that random motions of the probe molecules are restricted in a micellar medium. It can be seen that for each micellar system the fast rotational relaxation component has major contribution to the fluorescence depolarization than the slow component. The fact that the fast motions are responsible for the loss of anisotropy is evidenced by the values of time-zero anisotropy, $r_{\mathrm{o}}$ $<0.40$. It is known that the maximum possible value of $r_{\mathrm{o}}$ is $0.40 .^{72}$ The data in Tables $5 \mathrm{a}-5 \mathrm{c}$ show that fast as well as slow relaxation times along with the average relaxation time decrease with increasing concentration of urea in a given micelle. The variations of $\left\langle\tau_{\mathrm{r}}\right\rangle$ of C-480 with increasing concentration of urea in all three micellar systems are shown in Figure 14. The decreases in $\left\langle\tau_{\mathrm{r}}\right\rangle$ with increasing concentration of urea in a given micellar system is in the harmony of microviscosity of the systems. At a higher concentration of urea, the solvent molecules seep in the micelle. The urea molecules reduce the hydrophobic interaction between the surfactant molecules, which causes demicellization. As a result, the microviscosity of micelles decreases with increasing concentration of urea, which results in decrease of the rotational relaxation time. This phenomenon is also evidenced by almost continuous decrease in $r_{\mathrm{o}}$ value with increasing concentration of urea in each micellar system. Mukherjee et al. ${ }^{85}$ found that the rotational relaxation process of the probe molecules became faster when concentration of the urea is increased in F127 micelles. It is also pertinent to note that

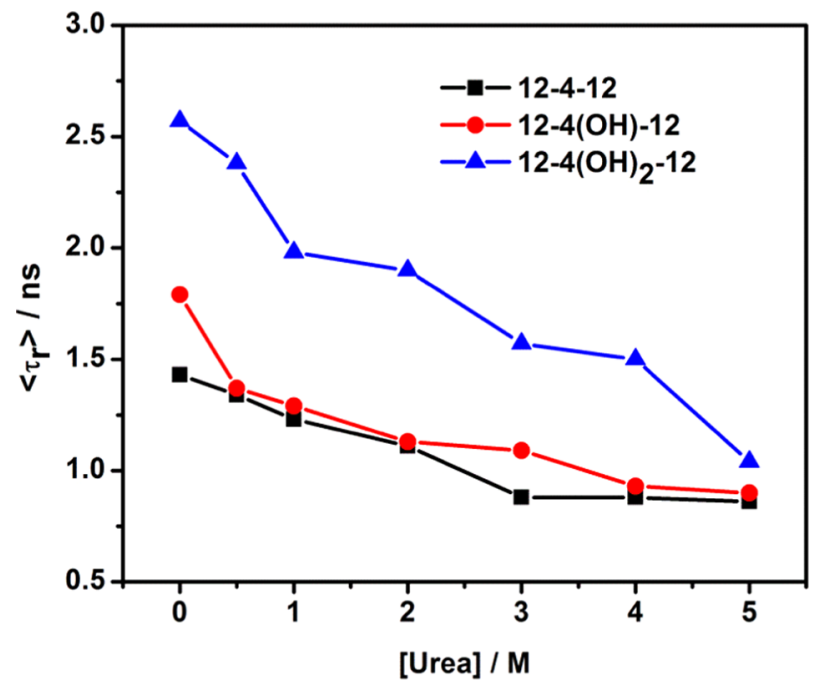

Figure 14. Average rotational relaxation time of C-480 in the presence of gemini surfactant and urea mixed systems.

at a given concentration of urea the increase in rotational relaxation time is according to the increasing order of microviscosity of micelles in the presence of more number of $-\mathrm{OH}$ groups starting from 0 to 2 .

From literature reports, it can be stated that the observed biexponential behavior of the anisotropy decay is mainly due to the different types of rotational motions. ${ }^{86,87}$ The two-step and wobbling-in-a-cone models ${ }^{87,88}$ are very suitable to explain this kind of bimodal behavior of anisotropy decay. According to the two-step model, the tumbling motion of the entire micelle $\left(\tau_{\mathrm{m}}\right)$ and the lateral diffusion $\left(\tau_{\mathrm{D}}\right)$ of the probe along the micelle surface contribute to the slow rotational relaxation $\left(\tau_{2 \mathrm{r}}\right)$. The time corresponding to the slow rotational relaxation is related to that of the overall motion of micelle as a whole and lateral diffusion as eq 12

$$
\frac{1}{\tau_{2 \mathrm{r}}}=\frac{1}{\tau_{\mathrm{D}}}+\frac{1}{\tau_{\mathrm{m}}}
$$


Table 6a. Hydrodynamic Diameter, Time for Overall Rotational Motion of the Micelle $\left(\tau_{\mathrm{m}}\right)$, Lateral Diffusion Time $\left(\tau_{\mathrm{D}}\right)$, Wobbling Motion Time $\left(\tau_{\mathrm{w}}\right)$, Wobbling Diffusion Coefficient $\left(D_{\mathrm{w}}\right)$, Cone Angle $\left(\boldsymbol{\theta}_{\mathrm{o}}\right)$, and Order Parameter $(|S|)$ Obtained from the Anisotropy Decays of C-480 in the Micelles of Gemini-A and Urea

\begin{tabular}{|c|c|c|c|c|c|c|c|c|}
\hline Gemini-A + urea & $\begin{array}{l}\text { hydrodynamic diameter } \\
(\mathrm{nm})\end{array}$ & $\tau_{\mathrm{w}}(\mathrm{ns})$ & $\tau_{\mathrm{m}}(\mathrm{ns})$ & $\tau_{\mathrm{D}}(\mathrm{ns})$ & $D_{\mathrm{w}} \times 10^{-8}\left(\mathrm{~s}^{-1}\right)$ & $\theta_{\mathrm{o}}(\mathrm{deg})$ & $|S|$ & $\theta_{\mathrm{o}}(\mathrm{deg})^{a}$ \\
\hline 0.0 & $1.25 \pm 0.06$ & $0.67 \pm 0.06$ & $221.46 \pm 0.53$ & $7.13 \pm 0.53$ & $4.93 \pm 0.77$ & $61.0 \pm 0.9$ & $0.36 \pm 0.03$ & $58.1 \pm 0.9$ \\
\hline 0.5 & $1.10 \pm 0.03$ & $0.74 \pm 0.07$ & $150.91 \pm 0.61$ & $7.79 \pm 0.61$ & $4.87 \pm 0.78$ & $63.7 \pm 0.9$ & $0.32 \pm 0.03$ & $53.1 \pm 0.9$ \\
\hline 1.0 & $1.38 \pm 0.07$ & $0.72 \pm 0.06$ & $297.99 \pm 0.63$ & $7.95 \pm 0.63$ & $5.44 \pm 0.69$ & $66.4 \pm 0.8$ & $0.28 \pm 0.02$ & $48.5 \pm 0.8$ \\
\hline 2.0 & $1.44 \pm 0.08$ & $0.74 \pm 0.07$ & $338.57 \pm 0.56$ & $7.11 \pm 0.56$ & $5.52 \pm 0.76$ & $67.8 \pm 0.8$ & $0.26 \pm 0.02$ & $46.1 \pm 0.8$ \\
\hline 3.0 & $2.07 \pm 0.12$ & $0.46 \pm 0.04$ & $1005.71 \pm 0.12$ & $1.73 \pm 0.12$ & $3.72 \pm 0.45$ & $43.9 \pm 0.7$ & $0.62 \pm 0.01$ & \\
\hline 4.0 & $2.13 \pm 0.14$ & $0.57 \pm 0.05$ & $1095.72 \pm 0.14$ & $2.09 \pm 0.14$ & $4.08 \pm 0.47$ & $51.2 \pm 0.6$ & $0.51 \pm 0.01$ & \\
\hline 5.0 & $2.14 \pm 0.15$ & $0.49 \pm 0.04$ & $1111.23 \pm 0.13$ & $1.96 \pm 0.13$ & $4.27 \pm 0.47$ & $48.6 \pm 0.6$ & $0.55 \pm 0.01$ & \\
\hline
\end{tabular}

Table $6 \mathrm{~b}$. Hydrodynamic Diameter, Time for Overall Rotational Motion of the Micelle $\left(\tau_{\mathrm{m}}\right)$, Lateral Diffusion Time $\left(\tau_{\mathrm{D}}\right)$, Wobbling Motion Time $\left(\tau_{\mathrm{w}}\right)$, Wobbling Diffusion Coefficient $\left(D_{\mathrm{w}}\right)$, Cone Angle $\left(\boldsymbol{\theta}_{\mathrm{o}}\right)$, and Order Parameter $(|S|)$ Obtained from the Anisotropy Decays of C-480 in the Micelles of Gemini-B and Urea

$\begin{array}{ccccccccc}\text { Gemini-B + urea } & \text { hydrodynamic diameter }(\mathrm{nm}) & \tau_{\mathrm{w}}(\mathrm{ns}) & \tau_{\mathrm{m}}(\mathrm{ns}) & \tau_{\mathrm{D}}(\mathrm{ns}) & D_{\mathrm{w}} \times 10^{-8}\left(\mathrm{~s}^{-1}\right) & \theta_{\mathrm{o}}(\mathrm{deg}) & |S| & \theta_{\mathrm{o}}(\mathrm{deg})^{a} \\ 0.0 & 1.30 \pm 0.06 & 0.71 \pm 0.07 & 249.11 \pm 0.98 & 13.86 \pm 0.98 & 5.29 \pm 0.75 & 65.0 \pm 0.8 & 0.30 \pm 0.02 & 50.8 \pm 0.8 \\ 0.5 & 1.50 \pm 0.07 & 0.74 \pm 0.07 & 382.68 \pm 0.87 & 10.68 \pm 0.87 & 5.52 \pm 0.81 & 67.8 \pm 0.8 & 0.26 \pm 0.02 & 46.1 \pm 0.8 \\ 1.0 & 1.28 \pm 0.06 & 0.68 \pm 0.06 & 237.79 \pm 0.65 & 8.25 \pm 0.65 & 5.53 \pm 0.74 & 65.0 \pm 0.8 & 0.30 \pm 0.02 & 50.8 \pm 0.8 \\ 2.0 & 1.38 \pm 0.06 & 0.71 \pm 0.05 & 297.99 \pm 0.44 & 5.39 \pm 0.44 & 4.96 \pm 0.58 & 63.0 \pm 0.8 & 0.33 \pm 0.02 & 54.3 \pm 0.8 \\ 3.0 & 1.78 \pm 0.08 & 0.75 \pm 0.06 & 639.47 \pm 0.43 & 5.13 \pm 0.43 & 4.81 \pm 0.51 & 63.7 \pm 0.6 & 0.32 \pm 0.01 & 53.1 \pm 0.7 \\ 4.0 & 1.58 \pm 0.08 & 0.61 \pm 0.05 & 447.23 \pm 0.15 & 2.52 \pm 0.15 & 4.21 \pm 0.45 & 53.8 \pm 0.6 & 0.47 \pm 0.01 & 75.8 \pm 0.9 \\ 5.0 & 1.34 \pm 0.07 & 0.68 \pm 0.05 & 272.82 \pm 0.14 & 2.27 \pm 0.14 & 3.78 \pm 0.37 & 53.8 \pm 0.6 & 0.47 \pm 0.01 & 75.8 \pm 0.9\end{array}$

${ }^{a} \theta_{\mathrm{o}}$ is calculated using the spinning-in-equatorial-band model.

Table 6c. Hydrodynamic Diameter, Time for Overall Rotational Motion of the Micelle $\left(\tau_{\mathrm{m}}\right)$, Lateral Diffusion Time $\left(\tau_{\mathrm{D}}\right)$, Wobbling Motion Time $\left(\tau_{\mathrm{w}}\right)$, Wobbling Diffusion Coefficient $\left(D_{\mathrm{w}}\right)$, Cone Angle $\left(\boldsymbol{\theta}_{\mathrm{o}}\right)$, and Order Parameter $(|S|)$ Obtained from the Anisotropy Decays of C-480 in the Micelles of Gemini-C and Urea

\begin{tabular}{ccccccccc} 
Gemini-C + urea & $\begin{array}{c}\text { hydrodynamic diameter } \\
(\mathrm{nm})\end{array}$ & \multicolumn{1}{c}{$\tau_{\mathrm{w}}(\mathrm{ns})$} & $\tau_{\mathrm{m}}(\mathrm{ns})$ & $\tau_{\mathrm{D}}(\mathrm{ns})$ & $D_{\mathrm{w}} \times 10^{-8}\left(\mathrm{~s}^{-1}\right)$ & $\theta_{\mathrm{o}}(\mathrm{deg})$ & $|S|$ & $\theta_{\mathrm{o}}(\mathrm{deg})^{a}$ \\
0.0 & $1.28 \pm 0.05$ & $0.81 \pm 0.08$ & $237.79 \pm 0.97$ & $13.55 \pm 0.97$ & $3.82 \pm 0.56$ & $59.0 \pm 0.8$ & $0.39 \pm 0.02$ & $62.0 \pm 0.9$ \\
0.5 & $1.28 \pm 0.05$ & $0.79 \pm 0.08$ & $237.79 \pm 0.89$ & $13.06 \pm 0.89$ & $4.09 \pm 0.59$ & $60.3 \pm 0.8$ & $0.37 \pm 0.02$ & $59.3 \pm 0.9$ \\
1.0 & $1.15 \pm 0.02$ & $0.84 \pm 0.08$ & $172.45 \pm 0.98$ & $13.69 \pm 0.98$ & $4.29 \pm 0.51$ & $63.7 \pm 0.7$ & $0.32 \pm 0.01$ & $53.1 \pm 0.7$ \\
2.0 & $1.22 \pm 0.04$ & $0.83 \pm 0.08$ & $205.89 \pm 0.99$ & $14.16 \pm 0.99$ & $4.53 \pm 0.64$ & $65.0 \pm 0.6$ & $0.30 \pm 0.02$ & $50.8 \pm 0.8$ \\
3.0 & $0.88 \pm 0.02$ & $0.81 \pm 0.07$ & $77.27 \pm 0.59$ & $7.93 \pm 0.59$ & $4.08 \pm 0.45$ & $61.0 \pm 0.7$ & $0.36 \pm 0.01$ & $58.1 \pm 0.7$ \\
4.0 & $1.05 \pm 0.03$ & $0.79 \pm 0.07$ & $131.26 \pm 0.63$ & $8.34 \pm 0.63$ & $4.46 \pm 0.59$ & $63.0 \pm 0.8$ & $0.33 \pm 0.02$ & $54.3 \pm 0.8$ \\
5.0 & $1.34 \pm 0.06$ & $0.49 \pm 0.05$ & $272.82 \pm 0.12$ & $1.91 \pm 0.12$ & $3.07 \pm 0.29$ & $41.1 \pm 0.6$ & $0.66 \pm 0.01$ &
\end{tabular}

${ }^{a} \theta_{\mathrm{o}}$ is calculated using the spinning-in-equatorial-band model.

The Debye-Stokes-Einstein equation is used to calculate $\tau_{\mathrm{m}}$ after estimating the hydrodynamic radii $\left(r_{h}\right)$ of micelle (Note 1, Supporting Information). The estimated $r_{\mathrm{h}}$ values for all three micellar media in the presence of urea are given in Tables 6a-6c. Figure S6 (Supporting Information) represents the size distribution plot for Gemini-B as a representative one. The $\tau_{\mathrm{m}}$ values at $298.15 \mathrm{~K}$ have been calculated using eq S1 (Supporting Information). After knowing the $\tau_{\mathrm{m}}$ values, the $\tau_{\mathrm{D}}$ values have been calculated using eq 12 . Both the $\tau_{\mathrm{m}}$ and $\tau_{\mathrm{D}}$ values are tabulated in Tables $6 \mathrm{a}-6 \mathrm{c}$. One can see that the $\tau_{\mathrm{D}}$ values (Tables $6 \mathrm{a}-6 \mathrm{c})$ are almost same as slow rotational relaxation time $\left(\tau_{2 \mathrm{r}}\right)$ (Tables 5a-5c). Thus, the lateral diffusion of the probe occurring along the surface of the micelle mainly contributes to the slow rotational relaxation. We have found that in case of Gemini-A there is an increasing tendency of the hydrodynamic radius of micelles with increasing concentration of urea. It infers that with increasing concentration of urea the micelles become less compact as the stabilization of micelle formation is reduced. As expected, the time constant for the tumbling motion of the micelle $\left(\tau_{\mathrm{m}}\right)$ increases with increasing size of micelles. However, we have not found any particular trend for hydrodynamic radii values of Gemini-B and Gemini- $\mathrm{C}$ with increasing concentration of urea, although there is a relationship between the size of the micelle and the $\tau_{\mathrm{m}}$ value. There is a diminishing tendency of $\tau_{\mathrm{D}}$ with increasing concentration of urea for a given micellar system. This trend is due to the decrease in the microviscosity of micelles with increasing concentration of urea. However, as $\tau_{\mathrm{D}}$ is related to $\tau_{\mathrm{m}}$, this correlation is also found to be comparatively better for Gemini-A than for Gemini-B and Gemini-C. The lateral diffusion of the probe molecule is much faster than the tumbling motion of the micelle as a whole. The time constants for the wobbling motion, $\tau_{\mathrm{w}}$, of the probe molecule C-480 in all of the micelles calculated using eq S2 (Note 2, Supporting Information) are also tabulated in Tables $6 a-6 c$. As the wobbling motion gives an account of the relaxation of local structure in a micelle, there is a decreasing tendency of the value of $\tau_{\mathrm{w}}$ with decreasing microviscosity of micelles.

The values of wobbling diffusion coefficient $\left(D_{\mathrm{w}}\right)$, order parameter $(|S|)$, and cone angle $\left(\theta_{\mathrm{o}}\right)$ have been calculated using eqs $\mathrm{S} 3-\mathrm{S} 5$, respectively, by applying the wobbling-in-a-cone 
model $^{50,87}$ (Note 3, Supporting Information) to obtain more information about the motion restriction of the probe molecules within the micelles. These values are tabulated in Tables 6a-6c. Quite large values of spatial restriction parameter $(|S|)$ show that the probe molecules are located in a restricted environment. The higher values of $\theta_{0}$ and lower values of $(|S|)$ at lower concentration range of urea could be due to the high microviscosity of micelles in this range. It is possible that the wobbling-in-a-cone model is not appropriate for micelles with high microviscosity. To solve this apparent problem, sometimes the spinning-in-equatorial-band model ${ }^{89,90}$ is used (Note 4, Supporting Information). We have applied the spinning-inequatorial-band model for micellar systems for which $|S|<0.5$, which is the requirement to apply this model. The calculated values of $\theta_{\mathrm{o}}$ after applying this model are also given in Tables $6 a-6 c$. Thus, possibly, the probe molecule is oriented in such a way that the emission moment remains perpendicular to the long axis like that in the spinning-in-equatorial-band model for micelles with high microviscosity. On the other hand, for micelles of comparatively lower microviscosity, the alignment of the C480 molecules would be such that the emission moment is parallel to the long axis like that is in the wobbling-in-a-cone model.

\section{CONCLUSIONS}

The effect of addition of urea in the aqueous micelles of all three cationic gemini surfactants with varying spacer groups on the micellization behavior of surfactants, solvation dynamics, and rotational relaxation of $\mathrm{C}-480$ has been studied. With increasing concentration of urea, the hydrophobic interactions between surfactant molecules are reduced due to lesser solvophobicity. This effect disfavors the formation of micelles, so $\mathrm{cmc}$ is increased with increasing concentration of urea. The most probable location of the C-480 molecules is the Stern layer of micelles. Solvation dynamics is found to be bimodal in nature. The average solvation time increases, reaches a maximum, and then decreases with increasing concentration of urea. It has been observed that the degree of counterion dissociation also increases, reaches a maximum, and then decreases with more and more addition of urea in the micellar solution. With increasing concentration of free counterions, the extent of clustering of water molecules is expected to be increased, resulting in longer solvation times. Thus, the rate of solvation process can be correlated with the degree of counterion dissociation. The presence of $-\mathrm{OH}$ group in the spacer group has an impact on the rate of solvation process. The $-\mathrm{OH}$ groups protect the water molecules from contact with the probe molecules through hydrogen bonding. As a result, the solvation process is slowed down. The microviscosity of micelles is decreased with increasing concentration of urea, as a result of which the rotational relaxation process becomes faster. At a given concentration of urea, the rotational relaxation process slows down with the introduction of the $-\mathrm{OH}$ group in the spacer group due to enhanced microviscosity of micelles. Rotational relaxation is bimodal in nature. The fast rotational relaxation component has major contribution to the fluorescence depolarization compared to the slow component. The lateral diffusion of the probe molecules along the surface of the micelle is mainly responsible for slow rotational relaxation. The tumbling motion of micelle as a whole is much slower than the lateral diffusion of the probe molecules. Wobbling motion also becomes faster with increasing concentration of urea as a result of the decreased microviscosity of micelles. The C-480 molecules might change their alignment in the micelles with changing microviscosity.

\section{EXPERIMENTAL SECTION}

4.1. Materials. Fluorescence probe $\mathrm{C}-480$ was procured from Exciton (laser grade) and used as received. Gemini surfactants were synthesized according the reported procedure. ${ }^{46,50-53}$ Synthesized gemini surfactants were recrystallized several times with a methanol and ethyl acetate mixture. Fourier transform infrared (FT-IR) and NMR spectroscopy methods were used to confirm the structure of the synthesized gemini surfactants given in the previous report. ${ }^{50} 1,6$-Diphenyl-1,3,5-hexatriene (DPH) was obtained from Aldrich Chemical Co. Extrapure urea was procured from SD Fine-Chem Limited, Mumbai, India. Aqueous solution of Ludox was obtained from Aldrich Chemical Co. This solution was used as a scatterer to record the lamp profile for time-correlated single-photon counting (TCSPC) measurements.

4.2. Methods. Solutions of pure gemini surfactants and gemini surfactants with urea at various concentrations were prepared in Milli-Q water obtained from Millipore water filtration system. The concentration of gemini surfactants in all of the measurements was fixed at $10 \mathrm{mM}$, which is quite higher than the $\mathrm{cmc}$ value of the respective gemini surfactants to make sure that the probe molecule completely solubilized in the micellar medium. ${ }^{50}$ A JASCO (Model V-650) UV-visible spectrophotometer was used to measure the absorption spectra of C-480 in pure solvents (UV-grade solvents) and micellar solutions. A HORIBA Jobin Yvon Fluoromax-4 scanning spectrofluorimeter was used to record to all of the fluorescence spectra. All of the fluorescence spectra were corrected for instrument sensitivity. For all of the fluorescence measurements, a slit width of $3 \mathrm{~nm}$ was kept for both the slits. For time-resolved measurements, the HORIBA Jobin Yvon Fluorocube-01-NL picosecond TCSPC experimental setup was used. A picosecond diode laser of wavelength $375 \mathrm{~nm}$ (NanoLED 375L, IBH, U.K.) was used as the light source. The details of the instruments is available in previous reports. ${ }^{50,51,91}$ The same setup was also used to analyze the time-resolved fluorescence anisotropy measurements. The time constants for solvation process have been estimated after constructing the TRES and calculating the solvent response function, $C(t)$, using the method of Fleming and Maroncelli, ${ }^{76}$ the details of which are available elsewhere. ${ }^{51}$ The time-resolved fluorescence anisotropy values, $r(t)$, have been calculated using eq 9. Hydrodynamic radii of micellar aggregates were measured using Zetasizer, model Nano ZS (ZEN 3600, Malvern Instruments, U.K.). Descriptions of dynamic light scattering (DLS) measurements are available in our earlier report. ${ }^{92}$ All samples were filtered before the measurements with a $0.22 \mu \mathrm{m}$ filter (Durapore, poly(vinylidene difluoride)). The wavelength of the laser light was $632.8 \mathrm{~nm}$, and the scattering angle was $173^{\circ}$. The corresponding $G$ function was considered carefully to judge the size distribution. The $\mathrm{cmc}$ values of all gemini surfactants in the absence and presence of different concentrations of urea were estimated by conductivity method using the Systronics conductivity meter 304. The conductivity cell with cell constant of $1 \mathrm{~cm}^{-1}$ was calibrated with the standard $\mathrm{KCl}$ solution of specific conductivity $1412 \mu \mathrm{S} \mathrm{cm}^{-1}$ prior to the measurement. Standard $\mathrm{KCl}$ solution was purchased from Merck, India. The ${ }^{1} \mathrm{H}$ NMR spectra were recorded with a Bruker Avance instrument $(400 \mathrm{MHz})$, and the FT-IR spectra were recorded in $\mathrm{ABB}$ Boman $\mathrm{MB} 300$ instrument. All measurements were carried out at $298.15 \pm 1 \mathrm{~K}$. 


\section{ASSOCIATED CONTENT}

\section{(5) Supporting Information}

The Supporting Information is available free of charge on the ACS Publications website at DOI: 10.1021/acsomega.7b01747. Absorption peak maxima $\left(\lambda_{\max }^{\mathrm{ab}}\right)$ and fluorescence peak maxima $\left(\lambda_{\max }^{\mathrm{fl}}\right)$ of C-480 in pure solvents and gemini surfactant-urea mixed systems (Table S1); fluorescence spectra of C-480 in different \% dioxane-water mixtures and variations in fluorescence intensity and fluorescence peak maxima of C-480 with increasing \% of water in dioxane-water mixtures (Figure $\mathrm{S} 1$ ); variation of the fluorescence energy at peak maximum $\left(\varepsilon_{\max }^{\mathrm{fl}}\right)$ of C-480 with $E_{\mathrm{T}}(30)$ of various dioxane-water mixtures (Figure S2); excited-state lifetimes of C-480 in the presence of pure gemini surfactants and with various concentrations of urea (Table S2); time-resolved fluorescence decays of C480 in Gemini-B and $-\mathrm{C}(10 \mathrm{mM})$ at $5 \mathrm{M}$ urea concentration (Figure S3); time-resolved emission spectra and decays of solvent response function, $C(t)$, of $C-480$ in Gemini-B and $-\mathrm{C}(10 \mathrm{mM})+X \mathrm{M}$ urea $(X=0,0.5,1,2,3$, 4 , and $5 \mathrm{M}$ ) (Figures S4 and S5); size distribution graph for the micelles of Gemini-B obtained from dynamic light scattering (DLS) measurement (Figure S6); and calculation of time corresponding to the rotational motion of the micelle as a whole $\left(\tau_{\mathrm{m}}\right)$, wobbling motion of the probe $\left(\tau_{\mathrm{w}}\right)$, wobbling diffusion coefficient $\left(D_{\mathrm{w}}\right)$, order parameter $|S|$, and cone angle $\left(\theta_{\mathrm{o}}\right)(\mathrm{PDF})$

(PDF)

\section{AUTHOR INFORMATION}

\section{Corresponding Authors}

*Email: sundar@hyderabad.bits-pilani.ac.in (G.S.).

*Email: sksaha@pilani.bits-pilani.ac.in, subitksaha@gmail.com. Tel.: +91-1596-515731. Fax: +91-1596-244183 (S.K.S.).

\section{ORCID}

Subit K. Saha: 0000-0001-8278-2912

\section{Present Address}

${ }^{\S}$ Department of School Education, Government Senior Secondary School (GSSS), Karsola, Haryana, 126101, India (S.).

\section{Notes}

The authors declare no competing financial interest.

\section{ACKNOWLEDGMENTS}

S.K.S. is grateful to the Council of Scientific and Industrial Research (CSIR) (01(2839)/16/EMR-II) for financial support and Department of Science and Technology (DST) FIST program, Government of India, and the University Grants Commission (UGC) for special assistance program [F.540/14/ DRS/2007(SAP-I)]. S.K. acknowledges the UGC-BSR and Birla Institute of Technology \& Science (BITS), Pilani, for financial assistance. S. acknowledges UGC, Government of India, for senior research fellowship. S.H. acknowledges UGC-DAE CSR, and R.A. acknowledges CSIR for fellowship.

\section{REFERENCES}

(1) Tanford, C. The hydrophobic effect and the organization of living matter. Science 1978, 200, 1012-1018.

(2) Israelachvili, J. N.; Marčelja, S.; Horn, R. G. Physical principles of membrane organization. Q. Rev. Biophys. 1980, 13, 121-200.
(3) Tanford, C. The Hydrophobic Effect: Formation of Micelles and Biological Membranes, 2nd ed.; John Wiley \& Sons: New York, 1980; pp $1-246$.

(4) Tanford, C. Amphiphile orientation: physical chemistry and biological function. Biochem. Soc. Trans. 1987, 15, 1S-7S PMID number (PMID:3328708).

(5) Garavito, R. M.; Ferguson-Miller, S. Detergents as tools in membrane biochemistry. J. Biol. Chem. 2001, 276, 32403-32406.

(6) Privé, G. G. Detergents for the stabilization and crystallization of membrane proteins. Methods 2007, 41, 388-397.

(7) Altschuler, M.; Heddens, D. K.; Diveley, R. R., Jr.; Kresheck, G. C. Plasmid DNA isolation utilizing a novel nonionic detergent. BioTechniques 1994, 17, 434-436 PMID number (PMID:7818891).

(8) Kresheck, G. C.; Hwang, J. Phase separation properties of several aqueous alkyldimethylphosphine oxide/phospholipid mixtures and their potential use for protein purification. Chem. Phys. Lipids 1995, 76, 193-199.

(9) Patra, S. K.; Alonso, A.; Goñi, F. M. Detergent solubilisation of phospholipid bilayers in the gel state: The role of polar and hydrophobic forces. Biochim. Biophys. Acta, Biomembr. 1998, 1373, 112-118.

(10) Kumbhakar, M.; Goel, T.; Mukherjee, T.; Pal, H. Effect of lithium chloride on the palisade layer of the Triton-X-100 micelle: Two sites for lithium ions as revealed by solvation and rotational dynamics studies. J. Phys. Chem. B 2005, 109, 18528-18534.

(11) Pramanik, R.; Sarkar, S.; Ghatak, C.; Rao, V. G.; Mandal, S.; Sarkar, N. Effects of 1-butyl-3-methyl imidazolium tetrafluoroborate ionic liquid on Triton X-100 aqueous micelles: Solvent and rotational relaxation studies. J. Phys. Chem. B 2011, 115, 6957-6963.

(12) Ruiz, C. C.; Sánchez, F. G. Effect of urea on aggregation behavior of Triton X-100 micellar solutions: A photophysical study. J. Colloid Interface Sci. 1994, 165, 110-115.

(13) Schick, M. J. Effect of electrolyte and urea on micelle formation. J. Phys. Chem. 1964, 68, 3585-3592.

(14) Causi, S.; De Lisi, R; Milioto, S.; Tirone, N. Dodecyltrimethylammonium bromide in water-urea mixtures: Volumes, heat capacities, and conductivities. J. Phys. Chem. 1991, 95, 5664-5673.

(15) Abu-Hamdiyyah, M.; Kumari, K. Solubilization tendency of 1alkanols and hydrophobic interaction in sodium lauryl sulfate in ordinary water, heavy water, and urea solutions. J. Phys. Chem. 1990, 94, $6445-6452$.

(16) Raghuraman, H.; Pradhan, S. K.; Chattopadhyay, A. Effect of urea on the organization and dynamics of Triton X-100 micelles: A fluorescence approach. J. Phys. Chem. B 2004, 108, 2489-2496.

(17) Almgren, M.; Swarup, S. Size of sodium dodecyl sulfate micelles in the presence of additives I. alcohols and other polar compounds. J. Colloid Interface Sci. 1983, 91, 256-266.

(18) Caponetti, E.; Causi, S.; De Lisi, R.; Floriano, M. A.; Milioto, S.; Triolo, R. Dodecyltrimethylammonium bromide in water-urea mixtures: Structure and energetics. J. Phys. Chem. 1992, 96, 4950-4960.

(19) Banères, J.-L.; Popot, J.-L.; Mouillac, B. New advances in production and functional folding of G-protein-coupled receptors. Trends Biotechnol. 2011, 29, 314-322.

(20) Buchanan, S. K. $\beta$-Barrel proteins from bacterial outer membranes: Structure, function and refolding. Curr. Opin. Struct. Biol. 1999, 9, 455-461.

(21) Stanley, A. M.; Fleming, K. G. The process of folding proteins into membranes: Challenges and progress. Arch. Biochem. Biophys. 2008, 469, 46-66.

(22) Fiedler, S.; Broecker, J.; Keller, S. Protein folding in membranes. Cell. Mol. Life Sci. 2010, 67, 1779-1798.

(23) Udho, E.; Jakes, K. S.; Buchanan, S. K.; James, K. J.; Jiang, X.; Klebba, P. E.; Finkelstein, A. Reconstitution of bacterial outer membrane TonB-dependent transporters in planar lipid bilayer membranes. Proc. Natl. Acad. Sci. U.S.A. 2009, 106, 21990-21995.

(24) Das, A.; Mukhopadhyay, C. Urea-mediated protein denaturation: A consensus view. J. Phys. Chem. B 2009, 113, 12816-12824.

(25) Stumpe, M. C.; Grubmüller, H. Aqueous urea solutions: Structure, energetics, and urea aggregation. J. Phys. Chem. B 2007, $111,6220-6228$. 
(26) Lee, M.-E.; van der Vegt, N. F. A. Does urea denature hydrophobic interactions? J. Am. Chem. Soc. 2006, 128, 4948-4949.

(27) Van der Vegt, N. F. A.; Trzesniak, D.; Kasumaj, B.; van Gunsteren, W. F. Energy-entropy compensation in the transfer of nonpolar solutes from water to cosolvent/water mixtures. ChemPhysChem 2004, 5, 144147.

(28) Finer, E. G.; Franks, F.; Tait, M. J. Nuclear magnetic resonance studies of aqueous urea solutions. J. Am. Chem. Soc. 1972, 94, 44244429 .

(29) Hoccart, X.; Turrell, G. Raman spectroscopic investigation of the dynamics of urea-water complexes. J. Chem. Phys. 1993, 99, 84988503

(30) Vanzi, F.; Madan, B.; Sharp, K. Effect of the protein denaturants urea and guanidinium on water structure: A structural and thermodynamic study. J. Am. Chem. Soc. 1998, 120, 10748-10753.

(31) Chitra, R.; Smith, P. E. Molecular dynamics simulations of the properties of cosolvent solutions. J. Phys. Chem. B 2000, 104, 58545864.

(32) Bennion, B. J.; Daggett, V. The molecular basis for the chemical denaturation of proteins by urea. Proc. Natl. Acad. Sci. U.S.A. 2003, 100, 5142-5147.

(33) Caflisch, A.; Karplus, M. Structural details of urea binding to barnase: A molecular dynamics analysis. Structure 1999, 7, 477.

(34) Hayashi, Y.; Katsumoto, Y.; Omori, S.; Kishii, N.; Yasuda, A. Liquid structure of the urea-water system studied by dielectric spectroscopy. J. Phys. Chem. B 2007, 111, 1076-1080.

(35) Bandyopadhyay, D.; Mohan, S.; Ghosh, S. K.; Choudhury, N. Molecular dynamics simulation of aqueous urea solution: is urea a structure breaker? J. Phys. Chem. B 2014, 118, 11757-11768.

(36) Bhattacharyya, K. Nature of biological water: A femtosecond study. Chem. Commun. 2008, 25, 2848-2857.

(37) Gratzel, M. Heterogeneous Photochemical Electron Transfer; CRC Press: Boca Raton, FL, 2017; pp 43-84.

(38) Rezus, Y. L. A.; Bakker, H. J. Effect of urea on the structural dynamics of water. Proc. Natl. Acad. Sci. U.S.A. 2006, 103, 18417-18420.

(39) Idrissi, A.; Sokolić, F.; Perera, A. A molecular dynamics study of the urea/water mixture. J. Chem. Phys. 2000, 112, 9479-9488.

(40) Kaatze, U.; Gerke, H.; Pottel, R. Dielectric relaxation in aqueous solutions of urea and some of its derivatives. J. Phys. Chem. 1986, 90, 5464-5469.

(41) Idrissi, A.; Bartolini, P.; Ricci, M.; Righini, R. Time resolved optical Kerr effect analysis of urea-water system. J. Chem. Phys. 2001, 114, 6774-6780.

(42) Reimers, J. R.; Watts, R. O.; Klein, M. L. Intermolecular potential functions and the properties of water. Chem. Phys. 1982, 64, 95-114.

(43) Gosting, L. J.; Akeley, D. F. A study of the diffusion of urea in water at $25^{\circ}$ with the Gouy Interference Method. J. Am. Chem. Soc. 1952, 74, 2058-2060.

(44) Sengupta, A.; Khade, R. V.; Hazra, P. How does the urea dynamics differ from water dynamics inside the reverse micelle? J. Phys. Chem. A 2011, 115, 10398-10407.

(45) Ruiz, C. C. Micelle formation and microenvironmental properties of sodium dodecyl sulfate in aqueous urea solutions. Colloids Surf., A 1999, 147, 349-357.

(46) Menger, F. M.; Keiper, J. S. Gemini surfactants. Angew. Chem., Int. Ed. 2000, 39, 1906-1920.

(47) Roy, S.; Dey, J. Effect of urea on self-organization of sodium N(11-acrylamidoundecanoyl)-L-valinate in water. J. Colloid Interface Sci. 2005, 290, 526-532.

(48) Broecker, J.; Keller, S. Impact of urea on detergent micelle properties. Langmuir 2013, 29, 8502-8510.

(49) Mahajan, R. K.; Kaur, R; Aswal, V. K. Effect of urea on the aggregation behavior of gemini surfactants and their mixed micelles with Pluronic L64. Colloids Surf., A 2013, 419, 61-68.

(50) Tiwari, A. K.; Sonu, K.; Saha, S. K. Effect of hydroxyl group substituted spacer group of cationic gemini surfactants on solvation dynamics and rotational relaxation of coumarin- 480 in aqueous micelles. J. Phys. Chem. B 2014, 118, 3582-3592.
(51) Sonu, K.; Kumari, S.; Saha, S. K. Effect of polymethylene spacer of cationic Gemini surfactants on solvation dynamics and rotational relaxation of coumarin 153 in aqueous micelles. J. Phys. Chem. B 2015, 119, 9751-9763.

(52) Sonu, K.; Tiwari, A. K.; Kumari, S.; Saha, S. K. Study on intramolecular charge transfer processes, solvation dynamics and rotational relaxation of coumarin 490 in reverse micelles of cationic gemini surfactant. RSC $A d v$. 2014, 4, 25210-25219.

(53) Sonu, K.; Kumari, S.; Saha, S. K. Solvation dynamics and rotational relaxation of coumarin 153 in mixed micelles of Triton X-100 and cationic gemini surfactants: Effect of composition and spacer chain length of gemini surfactants. Phys. Chem. Chem. Phys. 2016, 18, 15511563.

(54) Yip, R. W.; Wen, Y. X.; Szabo, A. G. Decay associated fluorescence spectra of Coumarin 1 and Coumarin 102: Evidence for a two-state solvation kinetics in organic solvents. J. Phys. Chem. 1993, 97, 1045810462.

(55) Jones, G.; Jackson, W. R.; Choi, C. Y.; Bergmark, W. R. Solvent effects on emission yield and lifetime for coumarin laser dyes. Requirements for a rotatory decay mechanism. J. Phys. Chem. 1985, $89,294-300$

(56) Chudoba, C.; Nibbering, E. T. J.; Elsaesser, T. Site-specific excited-state solute-solvent interactions probed by femtosecond vibrational spectroscopy. Phys. Rev. Lett. 1998, 81, No. 3010.

(57) Liu, Y.; Ding, J.; Shi, D.; Sun, J. Time-dependent density functional theory study on electronically excited states of coumarin 102 chromophore in aniline solvent: Reconsideration of the electronic excited-state hydrogen-bonding dynamics. J. Phys. Chem. A 2008, 112, 6244-6248.

(58) Palit, D. K.; Zhang, T.; Kumazaki, S.; Yoshihara, K. Hydrogenbond dynamics in the excited state of coumarin 102- aniline hydrogenbonded complex. J. Phys. Chem. A 2003, 107, 10798-10804.

(59) Zhao, G.-J.; Han, K.-L. Early time hydrogen-bonding dynamics of photoexcited coumarin 102 in hydrogen-donating solvents: Theoretical study. J. Phys. Chem. A 2007, 111, 2469-2474.

(60) Williams, R. J.; Phillips, J. N.; Mysels, K. J. The critical micelle concentration of sodium lauryl sulphate at $25^{\circ} \mathrm{C}$. Trans. Faraday Soc. 1955, 51, 728-737.

(61) Tiwari, A. K.; Sonu, K.; Sowmiya, M.; Saha, S. K. Micellization behavior of gemini surfactants with hydroxyl substituted spacers in water and water-organic solvent mixed media: The spacer effect. J. Mol. Liq. 2012, 167, 18-27.

(62) Wettig, S. D.; Nowak, P.; Verrall, R. E. Thermodynamic and aggregation properties of gemini surfactants with hydroxyl substituted spacers in aqueous solution. Langmuir 2002, 18, 5354-5359.

(63) de Moura, A. F.; Bernardino, K.; de Oliveira, O. V.; Freitas, L. C. G. Solvation of sodium octanoate micelles in concentrated urea solution studied by means of molecular dynamics simulations. J. Phys. Chem. B 2011, 115, 14582-14590.

(64) Paul, B. K.; Samanta, A.; Kar, S.; Guchhait, N. Evidence for excited state intramolecular charge transfer reaction in donor-acceptor molecule 5-(4-dimethylamino-phenyl)-penta-2, 4-dienoic acid methyl ester: Experimental and quantum chemical approach. J. Lumin. 2010, 130, 1258-1267.

(65) Grabowski, Z. R.; Rotkiewicz, K.; Rettig, W. Structural changes accompanying intramolecular electron transfer: Focus on twisted intramolecular charge-transfer states and structures. Chem. Rev. 2003, 103, 3899-4032.

(66) Maciejewski, A.; Kubicki, J.; Dobek, K. The origin of timeresolved emission spectra (TRES) changes of 4-aminophthalimide (4$\mathrm{AP}$ ) in SDS micelles. The role of the hydrogen bond between 4-AP and water present in micelles. J. Phys. Chem. B 2003, 107, 13986-13999.

(67) Mallick, A.; Purkayastha, P.; Chattopadhyay, N. Photoprocesses of excited molecules in confined liquid environments: An overview. J. Photochem. Photobiol., C 2007, 8, 109-127.

(68) Reichardt, C. Solvatochromic dyes as solvent polarity indicators. Chem. Rev. 1994, 94, 2319-2358.

(69) Mallick, A.; Haldar, B.; Maiti, S.; Bera, S. C.; Chattopadhyay, N. Photophysical study of 3-Acetyl-4-oxo-6, 7-dihydro-12 H-indolo [2,3-a] 
quinolizine in biomimetic reverse micellar nanocavities: A spectroscopic approach. J. Phys. Chem. B 2005, 109, 14675-14682.

(70) Sarkar, N.; Datta, A.; Das, S.; Bhattacharyya, K. Solvation dynamics of coumarin 480 in micelles. J. Phys. Chem. 1996, 100, 1548315486.

(71) Roy, S.; Mohanty, A.; Dey, J. Microviscosity of bilayer membranes of some $\mathrm{N}$-acylamino acid surfactants determined by fluorescence probe method. Chem. Phys. Lett. 2005, 414, 23-27.

(72) Lakowicz, J. R. Principles of Fluorescence Spectroscopy, 2nd ed.; Kluwer Academic: New York, 1999; pp 291-319.

(73) Shinitzky, M.; Barenholz, Y. Dynamics of the hydrocarbon layer in liposomes of lecithin and sphingomyelin containing dicetylphosphate. J. Biol. Chem. 1974, 249, 2652-2657.

(74) Kumari, S.; Aggrawal, R.; Sonu, K.; Halder, S.; Sundar, G.; Saha, S. K. Effect of hydrophobicity of tails and hydrophilicity of spacer group of cationic gemini surfactants on solvation dynamics and rotational relaxation of coumarin 480 in aqueous Micelles. ACS Omega 2017, 2, 5898-5910.

(75) Dutta, R.; Chowdhury, M.; Winnik, M. A. Solvent dependence of magnetic-field effect in a polymer-linked exciplex system. Polymer 1995, 36, 4445-4448.

(76) Maroncelli, M.; Fleming, G. R. Picosecond solvation dynamics of coumarin 153: The importance of molecular aspects of solvation. J. Chem. Phys. 1987, 86, 6221-6239.

(77) Asakawa, T.; Hashikawa, M.; Amada, K.; Miyagishi, S. Effect of urea on micelle formation of fluorocarbon surfactants. Langmuir 1995, 11, 2376-2379.

(78) Balasubramanian, S.; Bagchi, B. Slow solvation dynamics near an aqueous micellar surface. J. Phys. Chem. B 2001, 105, 12529-12533.

(79) Balasubramanian, S.; Pal, S.; Bagchi, B. Hydrogen-bond dynamics near a micellar surface: origin of the universal slow relaxation at complex aqueous interfaces. Phys. Rev. Lett. 2002, 89, No. 115505.

(80) Nandi, N.; Bagchi, B. Dielectric relaxation of biological water. J. Phys. Chem. B 1997, 101, 10954-10961.

(81) Kabir-ud-Din; Koya, P. A.; Khan, Z. A. Conductometric studies of micellization of gemini surfactant pentamethylene-1,5-bis (tetradecyldimethylammonium bromide) in water and water-organic solvent mixed media. J. Colloid Interface Sci. 2010, 342, 340-347.

(82) Solomons, T. W. G.; Fryhle, C. B. Organic Chemistry, 8th ed.; John Wiley \& Sons: Singapore, 2004; pp 262-264.

(83) Kumbhakar, M.; Goel, T.; Mukherjee, T.; Pal, H. Nature of the water molecules in the palisade layer of a Triton X-100 micelle in the presence of added salts: A solvation dynamics study. J. Phys. Chem. B 2005, 109, 14168-14174.

(84) Chakrabarty, D.; Chakraborty, A.; Seth, D.; Hazra, P.; Sarkar, N. Effect of alkyl chain length and size of the headgroups of the surfactant on solvent and rotational relaxation of Coumarin 480 in micelles and mixed micelles. J. Chem. Phys. 2005, 122, No. 184516.

(85) Anand, U.; Mukherjee, S. Microheterogeneity and microviscosity of F127 micelle: The counter effects of urea and temperature. Langmuir 2014, 30, 1012-1021.

(86) Kumbhakar, M.; Goel, T.; Mukherjee, T.; Pal, H. Role of micellar size and hydration on solvation dynamics: A temperature dependent study in Triton-X-100 and Brij-35 micelles. J. Phys. Chem. B 2004, 108, 19246-19254.

(87) Kinosita, K.; Kawato, S.; Ikegami, A. A theory of fluorescence polarization decay in membranes. Biophys. J. 1977, 20, 289-305.

(88) Chakrabarty, D.; Seth, D.; Chakraborty, A.; Sarkar, N. Dynamics of solvation and rotational relaxation of coumarin 153 in ionic liquid confined nanometer-sized microemulsions. J. Phys. Chem. B 2005, 109, $5753-5758$.

(89) Quitevis, E. L.; Marcus, A. H.; Fayer, M. D. Dynamics of ionic lipophilic probes in micelles: Picosecond fluorescence depolarization measurements. J. Phys. Chem. 1993, 97, 5762-5769.

(90) Johansson, L. B. A.; Niemi, A. Electronic energy transfer in anisotropic systems. 1. Octadecylrhodamine B in vesicles. J. Phys. Chem. 1987, 91, 3020-3023.

(91) Sowmiya, M.; Tiwari, A. K.; Sonu, K.; Eranna, G.; Sharma, A. K.; Saha, S. K. Study of the binding interactions of a hemicyanine dye with nanotubes of $\beta$-cyclodextrin and effect of a hofmeister series of potassium salts. J. Phys. Chem. C 2014, 118, 2735-2748.

(92) Muthusubramanian, S.; Tiwari, A. K.; Sonu, K.; Saha, S. K. A cationic surfactant-concentration dependent binding of a guest molecule with the nanotubes of $\beta$-cyclodextrin and its release from the nanotubular cavities. Soft Matter 2012, 8, 11072-11084. 